\title{
Methods Data Qualification Interim Report
}

R. Samuel Alessi

Tami Grimmett

LengVang

Dave McGrath

September 2010
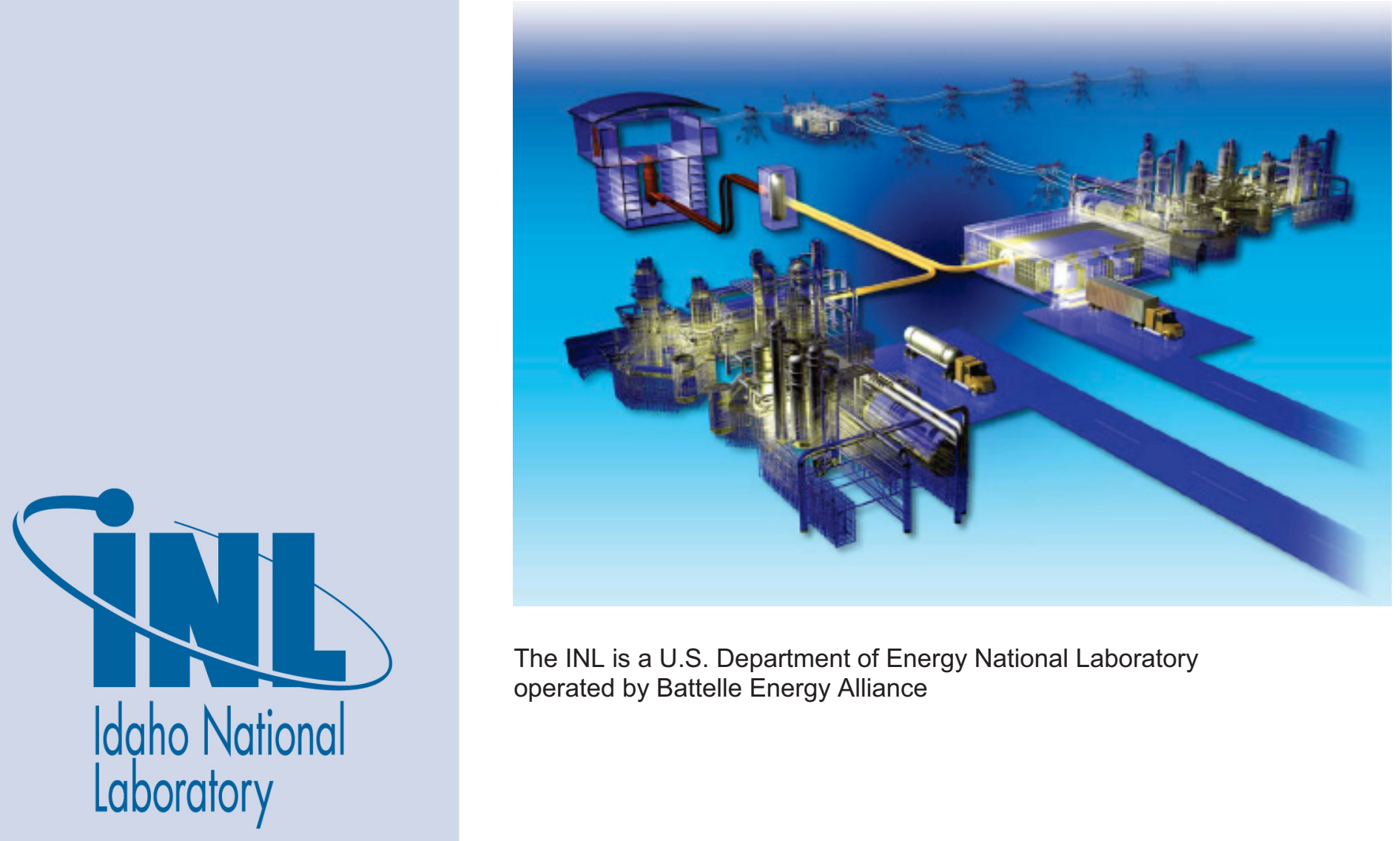

The INL is a U.S. Department of Energy National Laboratory operated by Battelle Energy Alliance 


\section{DISCLAIMER}

This information was prepared as an account of work sponsored by an agency of the U.S. Government. Neither the U.S. Government nor any agency thereof, nor any of their employees, makes any warranty, expressed or implied, or assumes any legal liability or responsibility for the accuracy, completeness, or usefulness, of any information, apparatus, product, or process disclosed, or represents that its use would not infringe privately owned rights. References herein to any specific commercial product, process, or service by trade name, trade mark, manufacturer, or otherwise, does not necessarily constitute or imply its endorsement, recommendation, or favoring by the U.S. Government or any agency thereof. The views and opinions of authors expressed herein do not necessarily state or reflect those of the U.S. Government or any agency thereof. 
INL/EXT-10-19798

\section{Methods Data Qualification Interim Report}

R. Samuel Alessi, Tami Grimmett, Leng Vang, and Dave McGrath

September 2010

Idaho National Laboratory

Next Generation Nuclear Plant Project

Idaho Falls, Idaho 83415

Prepared for the

U.S. Department of Energy

Office of Nuclear Energy

Under DOE Idaho Operations Office

Contract DE-AC07-05ID14517 
Next Generation Nuclear Plant Project

\section{Methods Data Qualification Interim Report}

INL/EXT-10-19798

September 2010

Approved by:
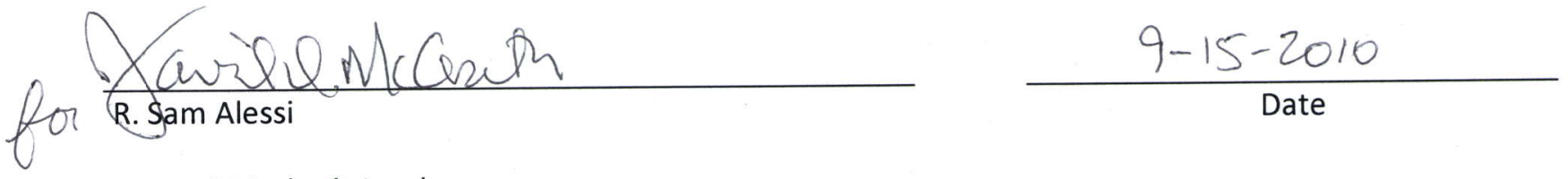

NDMAS Methods Lead
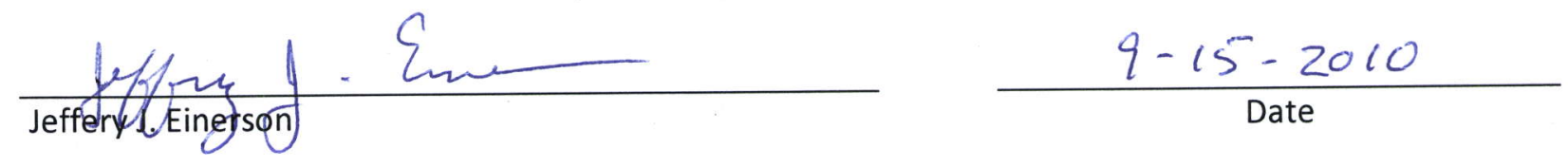

VHTR NDMAS Lead
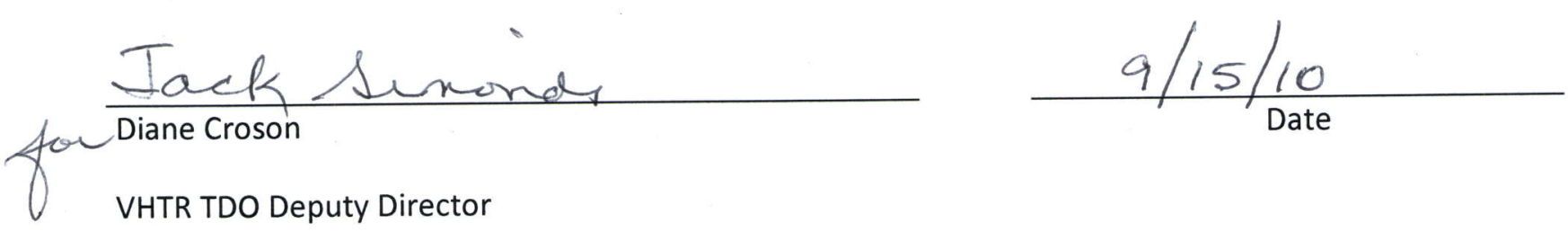


\section{EXECUTIVE SUMMARY}

The overall goal of the Next Generation Nuclear Plant (NGNP) Data Management and Analysis System (NDMAS) is to maintain data provenance ${ }^{\mathrm{a}}$ for the Next Generation Nuclear Plant (NGNP), including the Methods component of NGNP data. Multiple means are available to access data stored in NDMAS.

A Web portal environment allows users to access data, view the results of qualification tests, and view graphs and charts of various data attributes.

NDMAS also has methods for the management of the data output from very high temperature reactor simulation models and data generated from experiments designed to verify and validate the simulation codes. These simulation models represent the outcome of mathematical representations of very high temperature reactor components and systems. The Methods data management approaches described herein will handle data that arise from experiments, simulations, and external sources for the main purpose of facilitating parameter estimation and model verification and validation.

A model integration environment, named ModelCenter, is used to automate the storing of data from simulation model runs to the NDMAS repository. This approach does not adversely change the way computational scientists conduct their work. The method is to be used mainly to store the results of model runs that need to be preserved for auditing purposes or for display to the NDMAS Web portal.

This interim report discusses the current development activities of NDMAS for Methods data qualification.

a Provenance implies being able to reproduce the original and all transformations made to the data. 


\section{CONTENTS}

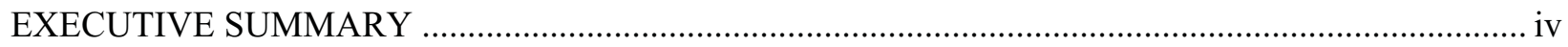

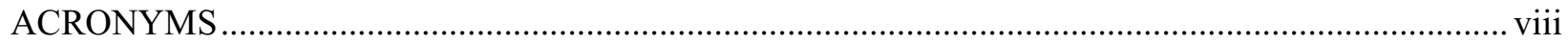

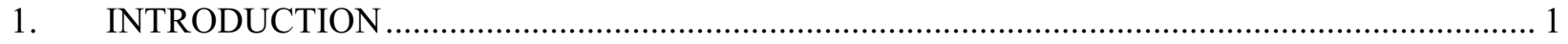

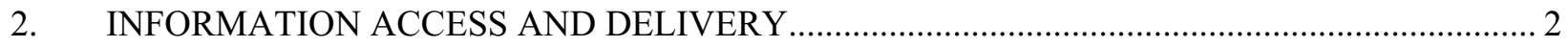

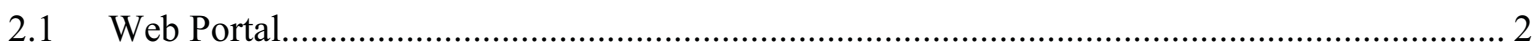

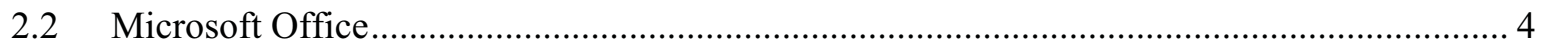

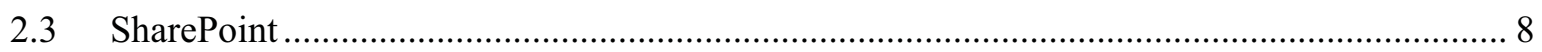

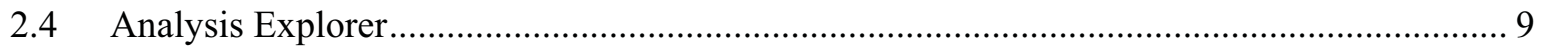

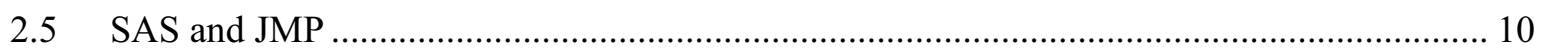

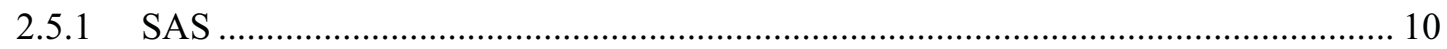

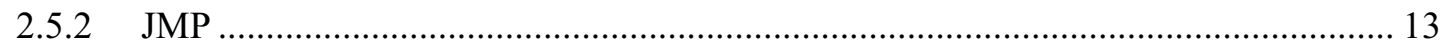

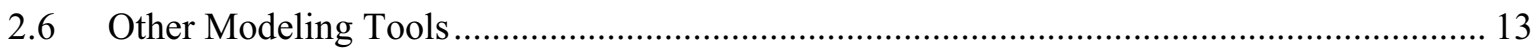

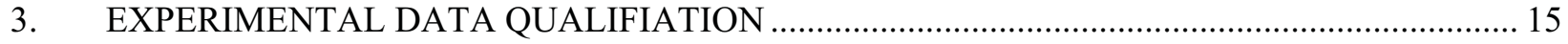

3.1 Lower Plenum Standard Problem ....................................................................................... 15

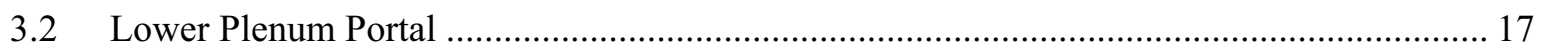

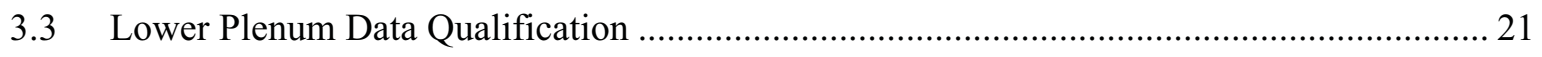

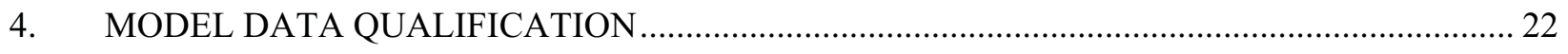

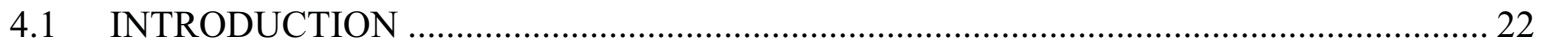

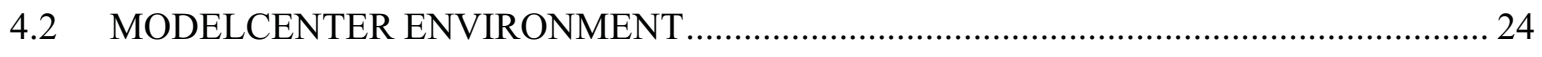

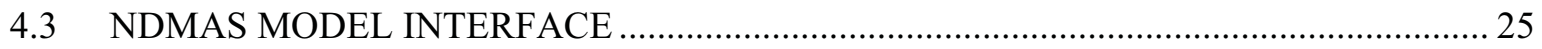

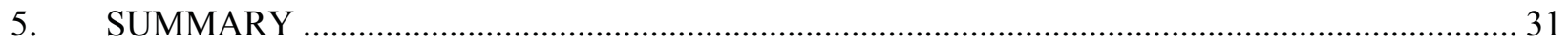

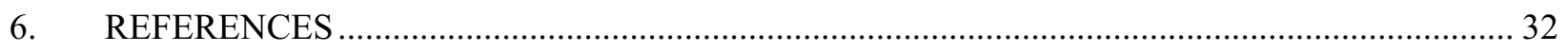

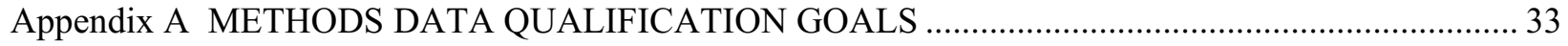

\section{FIGURES}

Figure 1. NDMAS Methods portal main page …...............................................................................

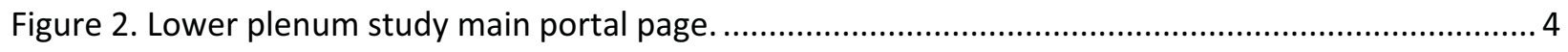

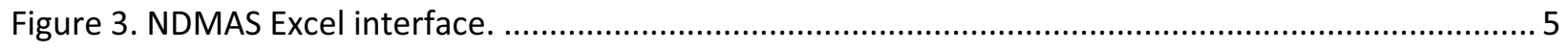

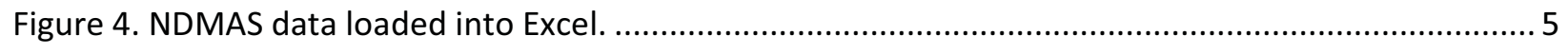

Figure 5. NDMAS SAS statistical routines available in Excel. .......................................................... 6 
Figure 6. NDMAS SAS Word interface showing a report loaded and the dialog to access NDMAS reports.

Figure 7. Methods SharePoint site. 8

Figure 8. NDMAS SharePoint Lower Plenum AutoCAD drawings of non-fluid experimental regions used to mask particle image velocimetry (PIV) data.

Figure 9. NDMAS Analysis Explorer displaying Lower Plenum study raw data files. 10

Figure 10. Contour plot created in JMP using Lower Plenum Jets 3 and 4 data. 13

Figure 11. Software and models used to satisfy the analysis requirements of the Methods Program (Schultz et al. 2005). 14

Figure 12. INL MIR facility. 15

Figure 13. Drawing of the scaled model used in the INL MIR facility to obtain flow data. Flow enters in from the top inlets and flows out toward the left rear; cylinders represent core support columns.

Figure 14. NDMAS Lower Plenum standard problem portal.

Figure 15. Lower Plenum portal for the composite of all the experimental data.

Figure 16. NDMAS report of composite Lower Plenum data.

Figure 17. Scalable graphs of Lower Plenum data along a vertical profile (y axis) for $z=-4 \mathrm{~mm}$ and $\mathrm{x}=91.01 \mathrm{~mm}$.

Figure 18. NDMAS data validation and qualification environment. Dialog shows point-and-click capability to test data for invalid values, missing values, duplication, and custom tests.

Figure 19. Cartoon showing the relationship between an individual computational scientist's work environment and ModelCenter data transfer to the NDMAS repository.

Figure 20. ModelCenter interface showing space nuclear integrated design model using Excel, a FEM mesher, Star CCM+, and Attila.

Figure 21. ModelCenter's Link Editor allows engineers to specify data passed from one application to the next when the model runs.

Figure 22. Analysis server running on a Windows platform.

Figure 23. NGNP Web portal. 26

Figure 24. Sample webpage that calls a ModelCenter model that runs a Matlab-based thermocouple prediction model and displays back the results. 
Figure 25. The ModelCenter thermocouple prediction model.

Figure 26. ModelCenter Matlab-plugin editor.

Figure 27. ModelCenter/SAS DataPipe plug-in for developing SQL queries connecting model

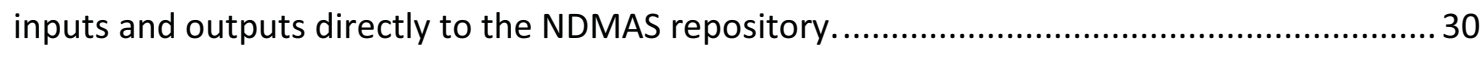

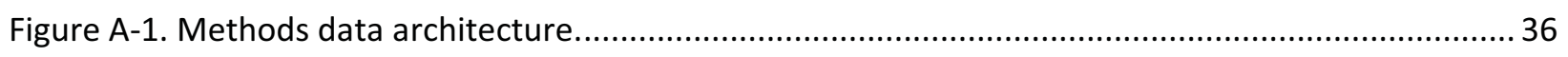

Figure A-2. NDMAS Version 1.1 data storage architecture. ............................................................ 39 


\section{ACRONYMS}

API application programming interface

CAD computer aided design

CFD computational fluid dynamics

CS computational scientists

DMZ Demilitarized Zone

DOE Department of Energy

HPC high performance computing

INET internal INL network

INL Idaho National Laboratory

MIR Matched-Index-of-Refraction

NASA National Aeronautics and Space Administration

NDMAS NGNP Data Management and Analysis System

NGNP Next Generation Nuclear Plant

NREL National Renewable Energy Laboratory

PIV particle image velocimetry

$\mathrm{V} \& \mathrm{~V} \quad$ verification and validation

VHTR very high temperature reactor 


\section{Methods Data Qualification Interim Report \\ 1. INTRODUCTION}

Data generated by Next Generation Nuclear Plant (NGNP) experiments and models are being captured by NGNP Data Management and Analysis System (NDMAS). The system is designed to provide the capability to capture, scrutinize, warehouse, and deliver both experimental and simulated (model) data from all sources relevant to the NGNP Project. A primary goal of the NDMAS system is to maintain data provenance of NGNP data. "Data provenance relative to" NDMAS records means: "where a piece of data came from and the process by which it arrived in the database ..." Provenance is becoming increasingly important, especially in scientific databases where understanding provenance is crucial to the accuracy and currency of data" (Buenman et al., 2001) and makes that information available for auditing purposes. NDMAS adheres to NGNP, Department of Energy (DOE), and NQA-1 quality standards (Krusch 2009, 2010).

This report provides information on how Methods data can be accessed by users and what tools are available for analysis. The main tool used to access Methods data is through the NDMAS Web portal - an implementation of SAS ${ }^{b}$ Web portal technology. Data can be also be accessed through Microsoft Office products. For example, team-working documents are shared through the NDMAS' Microsoft SharePoint portal (a simulation data management tool entitled Analysis Explorer), SAS analysis tools, and also user preferred analysis tools through a model integration environment named ModelCenter. ${ }^{\mathrm{c}}$ Together these products offer users multiple means to access and analyze Methods data. NDMAS is designed to allow researchers to work in their personally configured work environments while easily transferring data to the NDMAS NQA-1 data repository.

Lower plenum standard problem data is presented as it can be accessed through NDMAS. This single experiment (one of 200+ planned by the Methods Program) collected approximately 4 million measurements. The NDMAS Web portal currently offers access to the Lower Plenum data as a drillable data grid, associated graphs, and vector flow graphs. This NDMAS portal also allows users to customize the presentation of the data by applying filters, conditional highlighting, and calculations of new quantities.

NDMAS can capture data generated by NGNP models that perform simulations of reactor components and systems. Data derived from models complicate maintenance of data provenance, since provenance is degraded when data is downloaded from a controlled database to personal computers (Excel, text, binary, etc.), fed as input into models, generated as results (Excel, text, binary, etc.), and then uploaded back into a database repository. Provenance in such an environment, if done at all, requires time-consuming hand entry methods. NDMAS has developed tools for maintaining provenance automatically during model execution, or data can be manually uploaded to NDMAS.

This report presents the interim status of NDMAS applied to Methods' needs. Work is ongoing to incorporate additional Methods experimental data and model results into the NDMAS repository and Web delivery portal.

\footnotetext{
a Provenance implies being able to reproduce the original and all transformations made to the data.

b. "SAS" is a copyright of SAS Institute, Inc. SAS is a suite of commercial off-the-shelf statistical analysis software servers and client applications utilized by the NGNP project.

c. "ModelCenter" is a model integration server technology developed by Phoenix Integration, Inc.
} 


\section{INFORMATION ACCESS AND DELIVERY}

NDMAS provides multiple means for users ${ }^{\mathrm{d}}$ to access NGNP information. The main delivery mechanism is a Web portal accessed by most popular Web browsers. The Web portal allows data access (e.g. viewing and download), the viewing of charts, graphs, and reports, and report customization. Microsoft Office ${ }^{\mathrm{e}}$ tools (Word, PowerPoint, and Excel) provide access to NDMAS information. Updating of graphs, charts, and data grids embedded into Word reports or PowerPoint presentations occur automatically when source server data changes. SAS also integrates its functions and tools within the Microsoft Office toolbars to bring the statistical power of SAS to the familiar Microsoft Excel interface.

NDMAS provides repositories for project metadata and supporting information. NDMAS uses Microsoft SharePoint to support the storage of a variety of documents in addition to functioning as a project collaboration environment. Another approach, Analysis Explorer, provides a means to manage information that supports model development and simulation experiments.

NDMAS includes a statistical analysis environment that is available to all users within the Idaho National Laboratory (INL) firewall. Two products, SAS and JMP, ${ }^{f}$ represent a complete statistical capability tightly integrated with the NDMAS information delivery environment. These tools provide users with the ability to deliver analysis reports directly to the Web portal without the help NDMAS staff.

NDMAS supports integration of other modeling tools through a model integration environment that coupled to the NDMAS database repository. This capability allows computational scientists to conduct simulation experiments using individually preferred tools, while reading and writing data directly to the NDMAS repository without manual upload/download. This approach automatically ensures the provenance and configuration management of simulation experimental data for auditing purposes.

Together these approaches provide means to support a broad set of users of NGNP information. The remainder of this section will describe each information access and delivery option. All the tools discussed require user authentication and apply required security measures. Contact the NDMAS operations lead ${ }^{\mathrm{g}}$ for information on how to gain access to NDMAS and any of the tools discussed in this report.

\subsection{Web Portal}

The NDMAS Web portal is the primary means for delivering NGNP data. The portal provides nonINL users with access to NDMAS Methods data via a Web URL link.

Figure 1 shows the main interface to the Method's portion of the NDMAS portal that provides project information, contact information and links to more detailed project documents. The left panel is an expandable tree structure that allows users to access Methods as well as other NGNP data and NDMAS reports. The Methods' portals have been organized by Validation Matrix, Facilities, Experiments, and Modeling. Users hover the mouse pointer over a label in the left panel tree to display more information about that portal page.

d. NDMAS supports four main user groups including, managers, experimentalists, computational scientists, and auditors.

e. The Office interface is not available outside the INL firewall. Only WEB access is available to external users.

f. "JMP" is a subsidiary company of SAS Institute, Inc whose flagship product (also called JMP) is a statistical visualization software tool.

g. Kirk Fitzgerald (kirk.fitzgerald@inl.gov), NDMAS Operations Lead. 


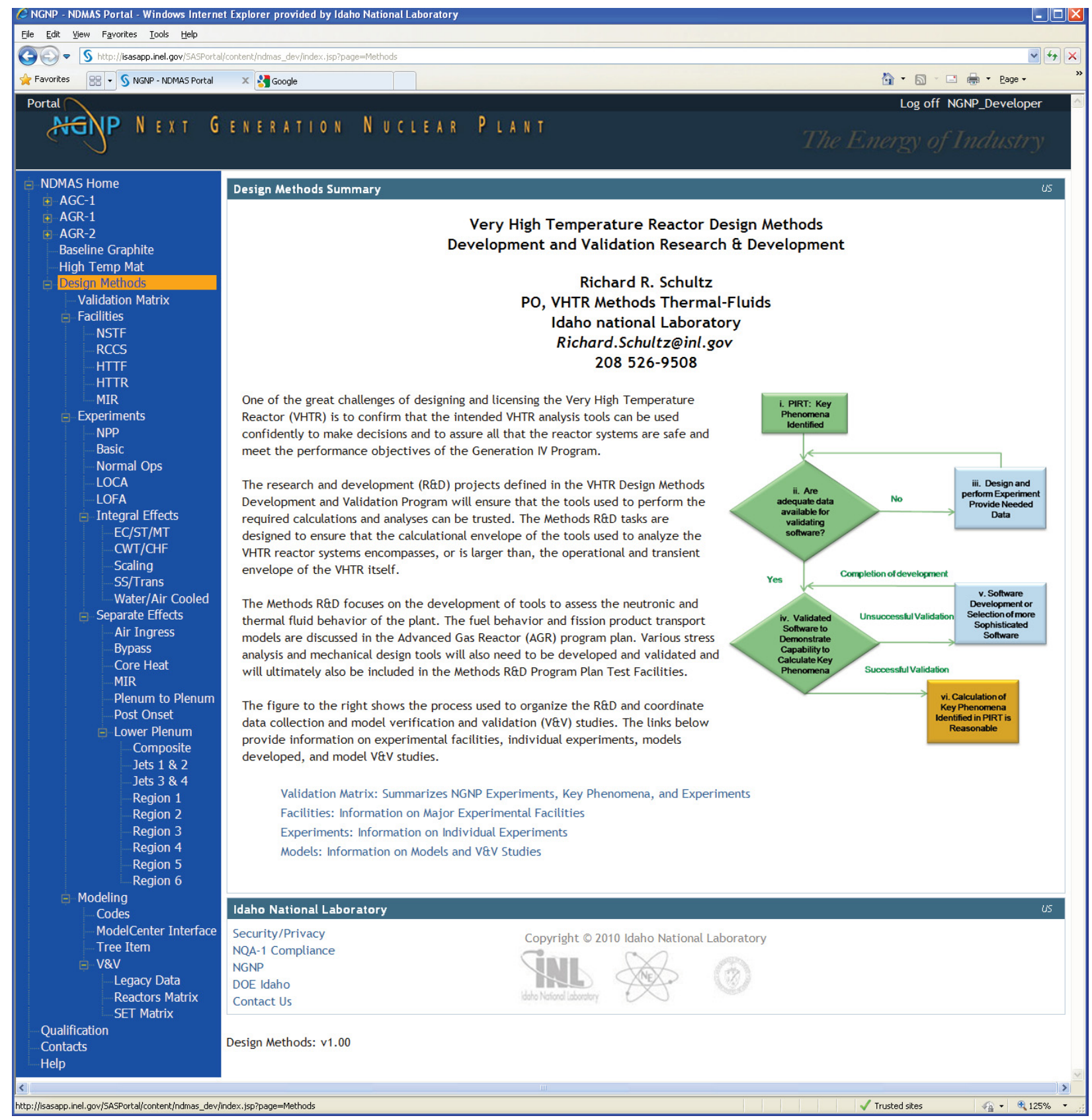

Figure 1. NDMAS Methods portal main page

Currently, Separate Effects, Lower Plenum Effects, Lower Plenum Study, and the ModelCenter Interface are populated. Later sections of this report discuss each of these sections.

Figure 2 shows the main portal page for the Lower Plenum study. A project description and contact information for the studies' principle investigator are provided. Drawings are displayed that help the user orient to the geometry of the study, as well as provide links to the SharePoint repository where reports that describe the project are stored.

A full explanation of the Lower Plenum data will be given in Section 3 of this report. 


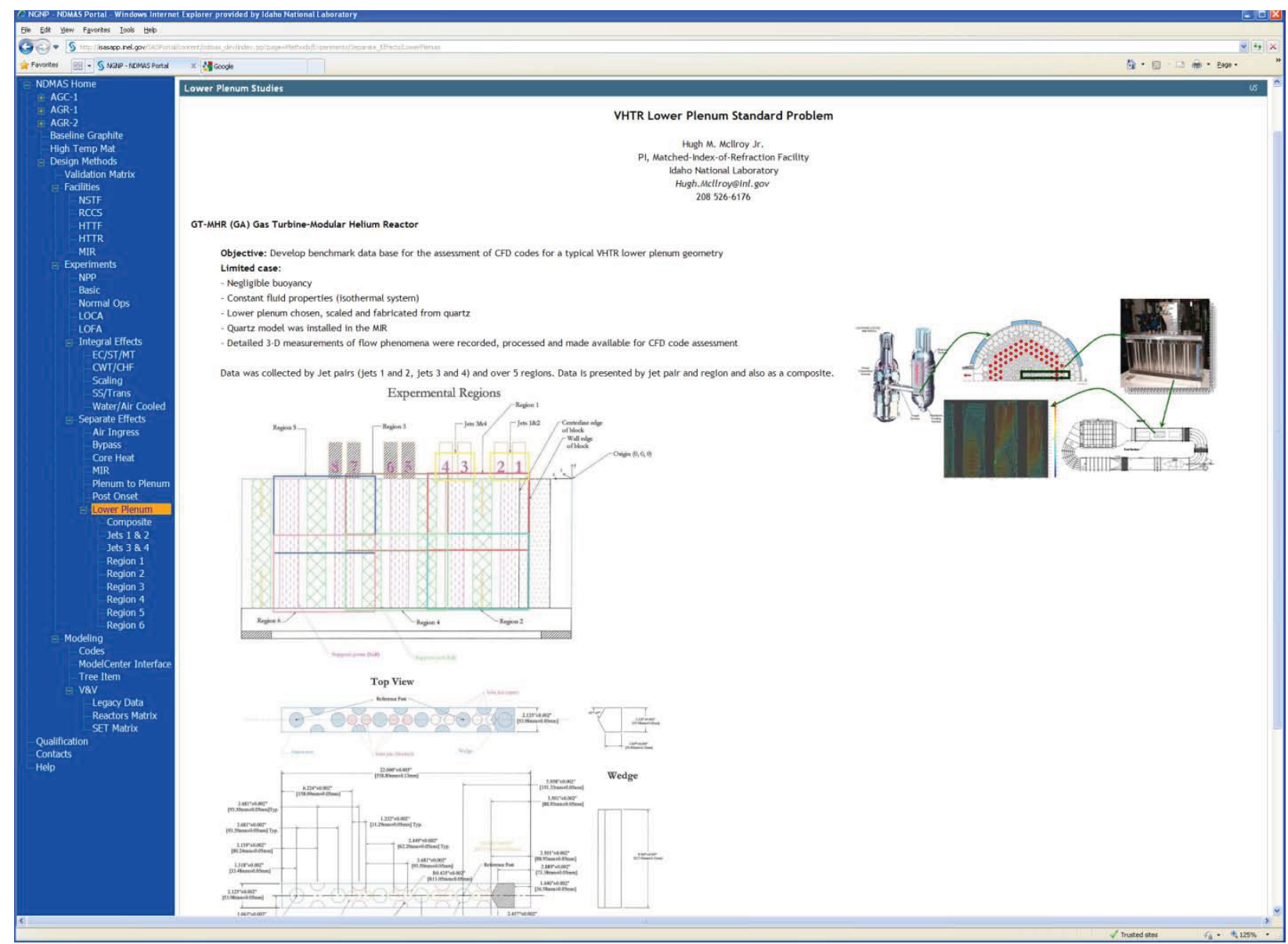

Figure 2. Lower plenum study main portal page.

\subsection{Microsoft Office}

NDMAS provides access to NGNP information through Microsoft Office products. Figure 3 shows the SAS tab in Microsoft Excel. ${ }^{\text {h }}$ The dialog box displays NDMAS data files located in the Lower Plenum NDMAS data library.

h. Contact Kirk Fitzgerald (kirk.fitzgerald@inl.gov), NDMAS Operations Lead for help installing the SAS Office Plugin. 


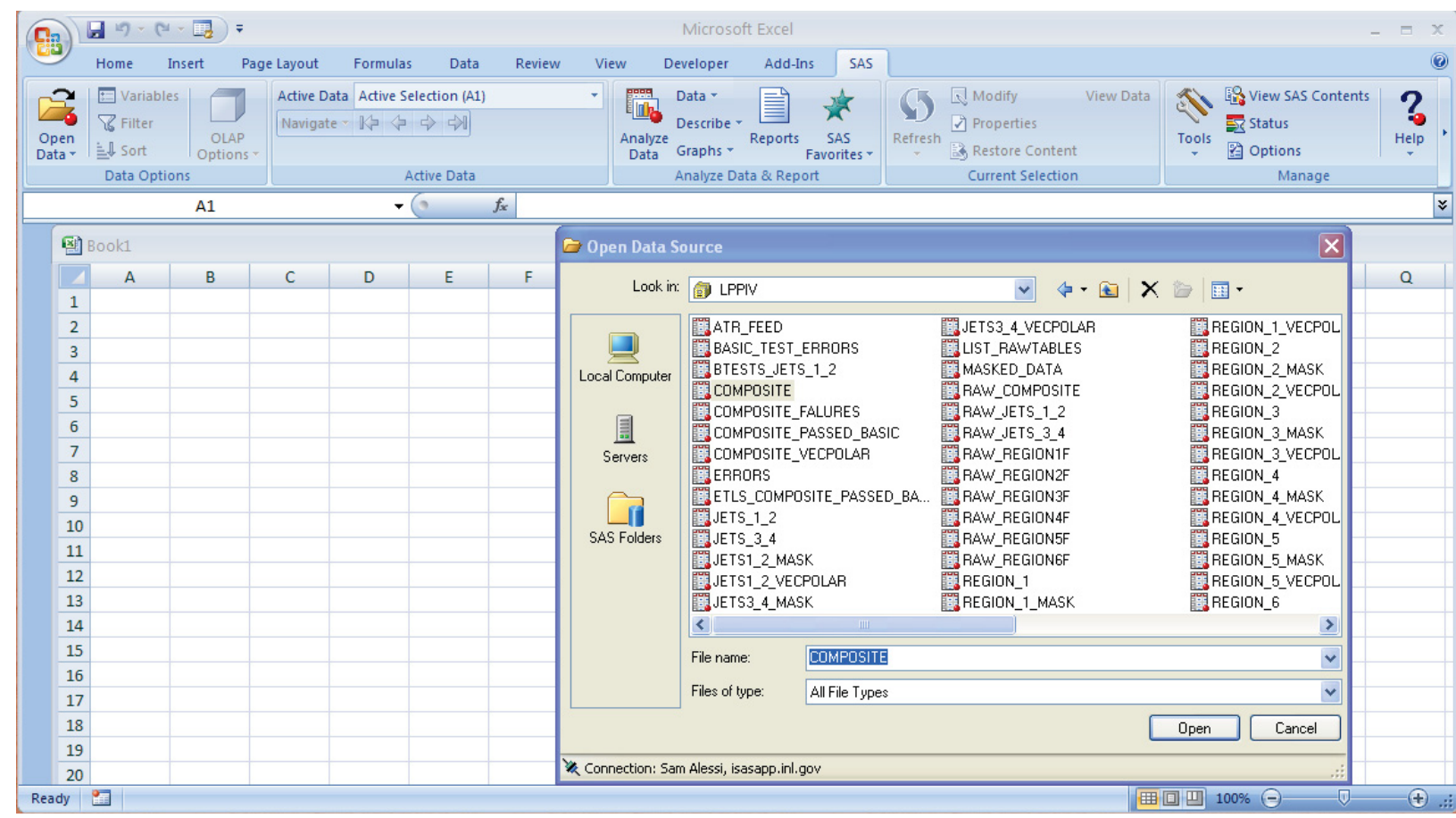

Figure 3. NDMAS Excel interface.

Figure 4 shows the data loaded into Excel. Utilizing SAS enables Excel to load and parse large data sets (greater than Excel's 24,000 row limit). SAS analytical and statistical routines are available within a SAS toolbar embedded into Excel.

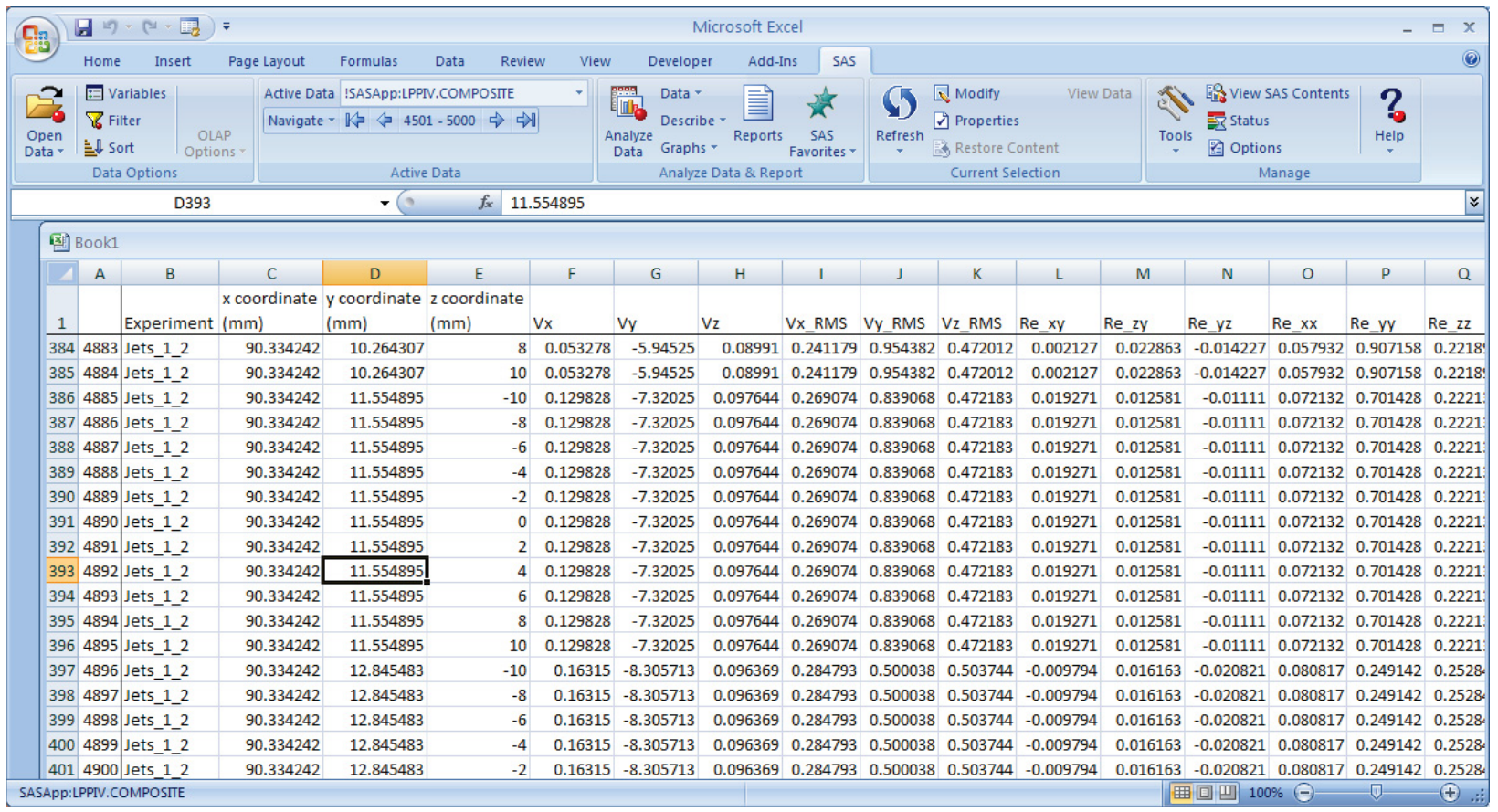

Figure 4. NDMAS data loaded into Excel.

All the familiar Excel functions remain available to analyze this data. Figure 5 shows a foreground dialog box that provides multiple choices for using SAS routines to transform and model the data. The 
example shows choices for different forms of regression analysis, though many other choices of statistical algorithms are available.

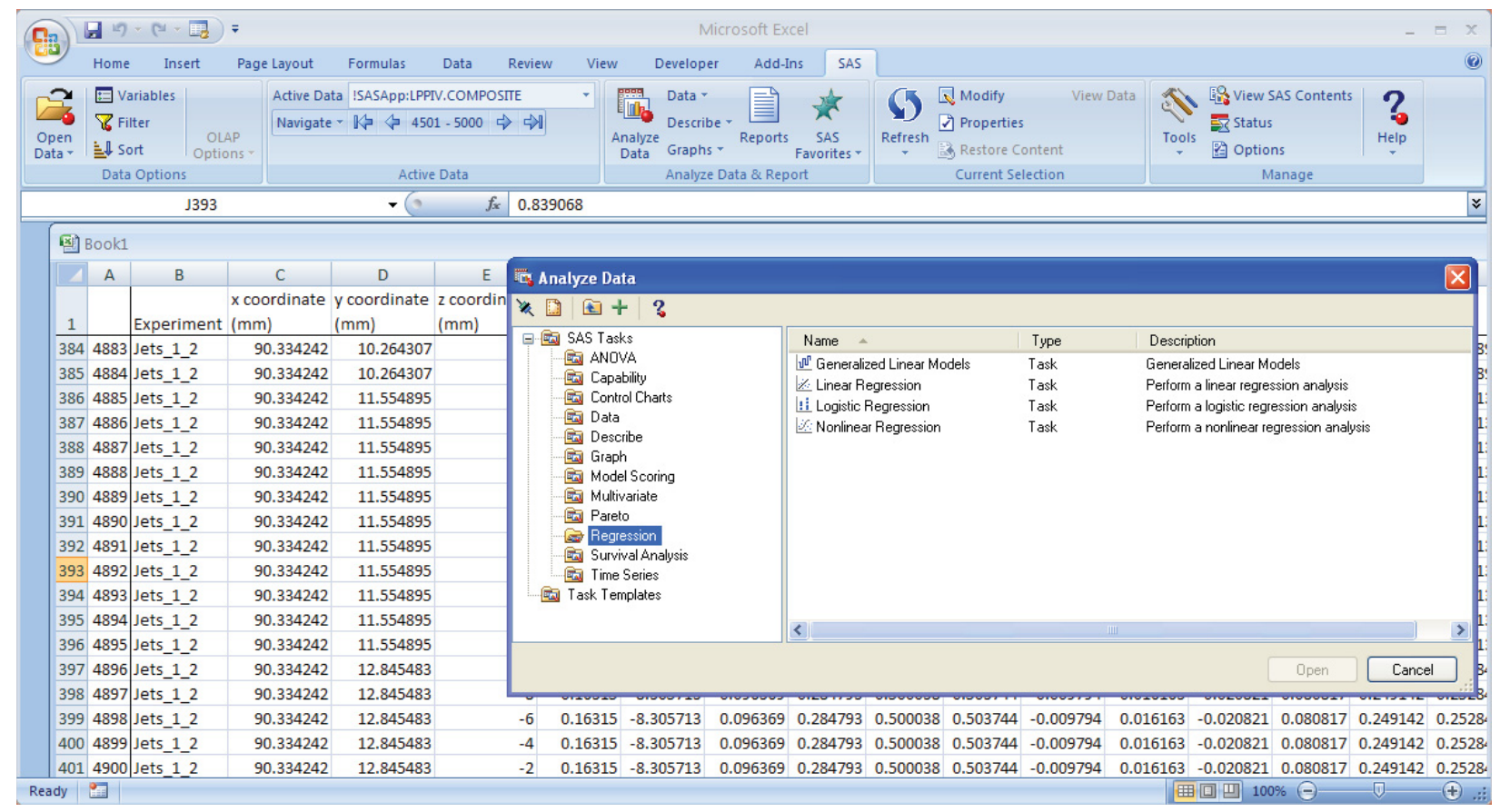

Figure 5. NDMAS SAS statistical routines available in Excel.

Section 2.5 covers SAS statistical routines.

The SAS/Excel interface also allows users to publish the reports and analysis generated in Excel to the NDMAS library. These reports are available to authenticated users within the NDMAS Web portal.

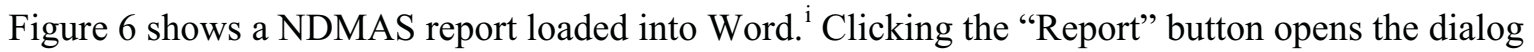
box shown. The displayed reports contain the same list of reports discussed previously in the Web portal section. Furthermore, all these reports receive data from the same NDMAS data tables so data tables and graphs will render the same data whether viewed from the website or from within Word. Changes and additions to the data will be automatically included within these reports such that Methods reports that have embedded NDMAS reports simply refresh to update tables, graphs, and charts: no manual cutting/pasting, uploading/downloading required. This approach should reduce errors introduced by manual steps. It should also allow report writers to spend more time on technical issues and less on the logistics of file manipulation.

The Word SAS toolbar also allows users to access data directly and to analyze data and create graphs in Excel. Users publish reports created in Word to NDMAS and then post those reports to the NDMAS Web portal.

i. The PowerPoint interface is similar to the Word interface to NDMAS reports and data and is therefore not shown. 


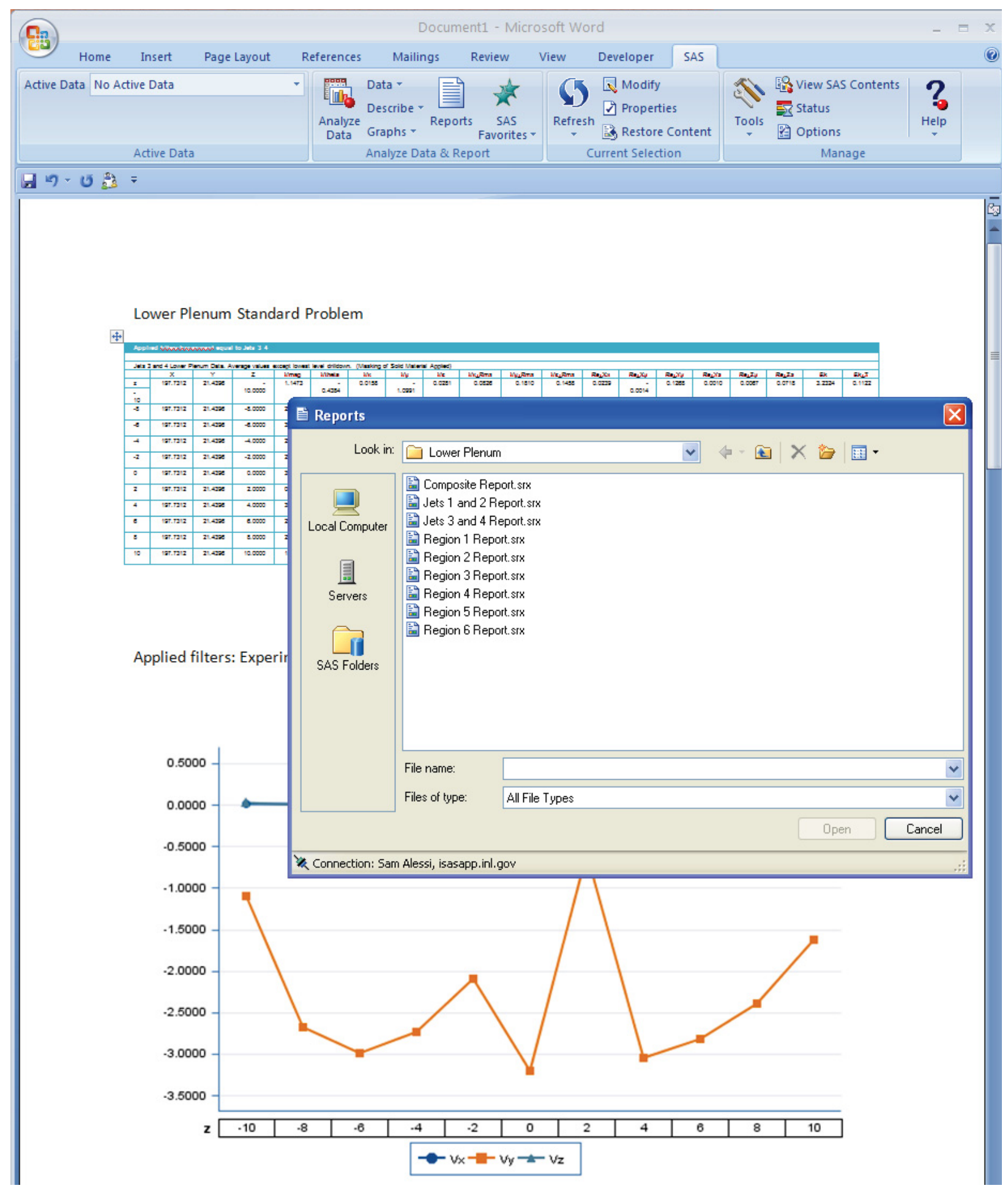

Figure 6. NDMAS SAS Word interface showing a report loaded and the dialog to access NDMAS reports. 


\subsection{SharePoint}

SharePoint is a Microsoft developed Web-based content management system used by the NDMAS team, which provides numerous project collaboration features including software development and project management tools. A full discussion of SharePoint features is beyond the scope of this document ${ }^{j}$. This section covers some of the main features currently used for Methods data qualification.

Figure 7 shows the main Methods SharePoint portal. ${ }^{\mathrm{k}}$ The main screen has three portlets currently installed: Announcements, Calendar, and Links. Two document areas exist: one for Methods documents and a second that pertains to the engineering of the NDMAS Methods capabilities. SharePoint facilitates management of issues, risks, deliverables, and tasks among the team. On-line team discussions are available, as well as many other capabilities not shown.

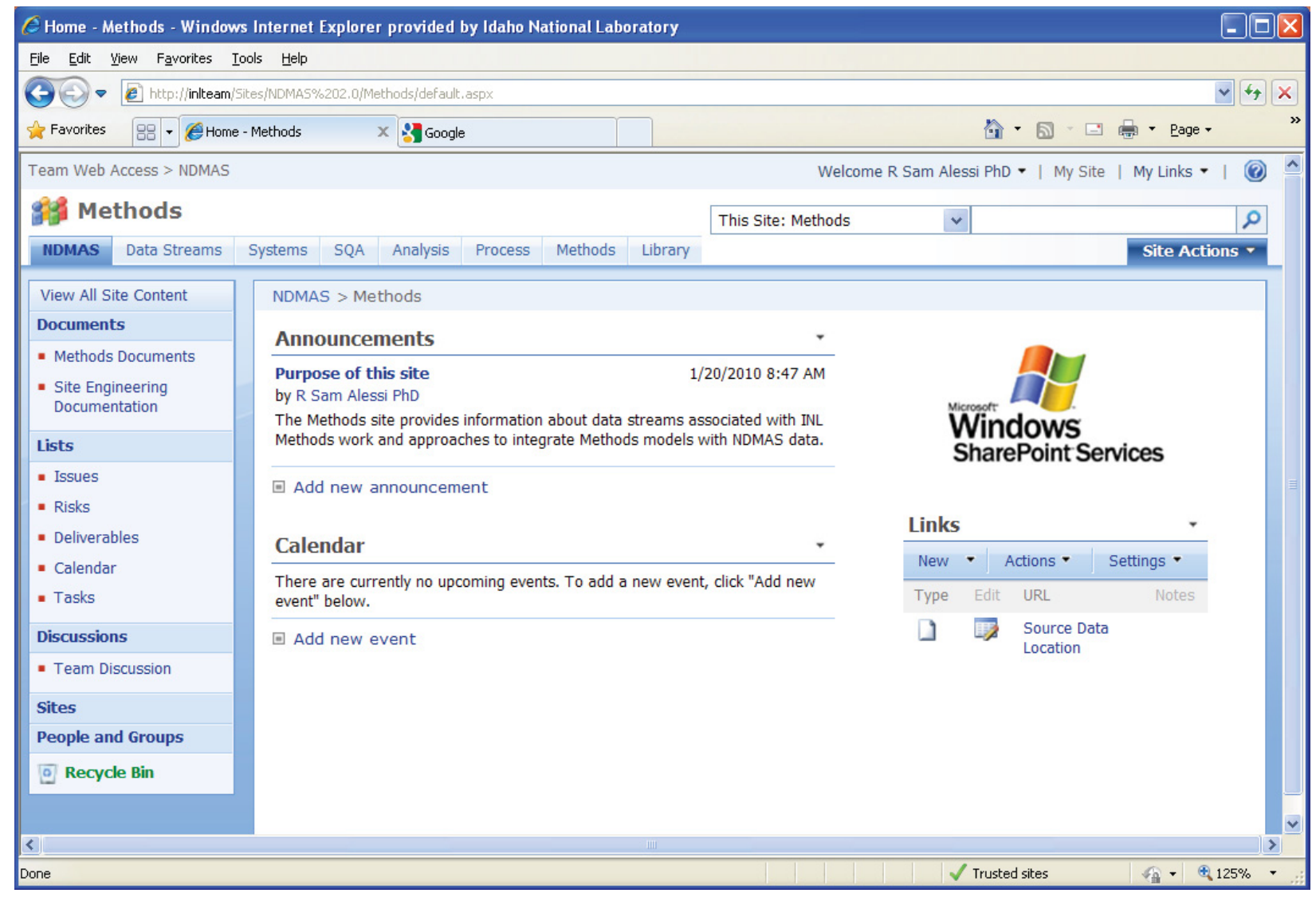

Figure 7. Methods SharePoint site.

To highlight a current use of the Methods SharePoint site, Figure 8 shows a drill down into the Methods Documents section to the AutoCAD files section uploaded by Hugh McIlroy, the Lower Plenum Standard Problem Principle Investigator. These computer aided design (CAD) drawings document the correction of the Lower Plenum experimental data as it moved into the NDMAS repository. These files are under version control and permission to access or change these files is restricted.

j. Contact Sam Alessi (rsam.alessi@inl.gov) if you are interested in using SharePoint tools for Methods research and model development.

k. Contact Kirk Fitzgerald (kirk.fitzgerald@inl.gov), NDMAS Operations Lead for access privileges. 


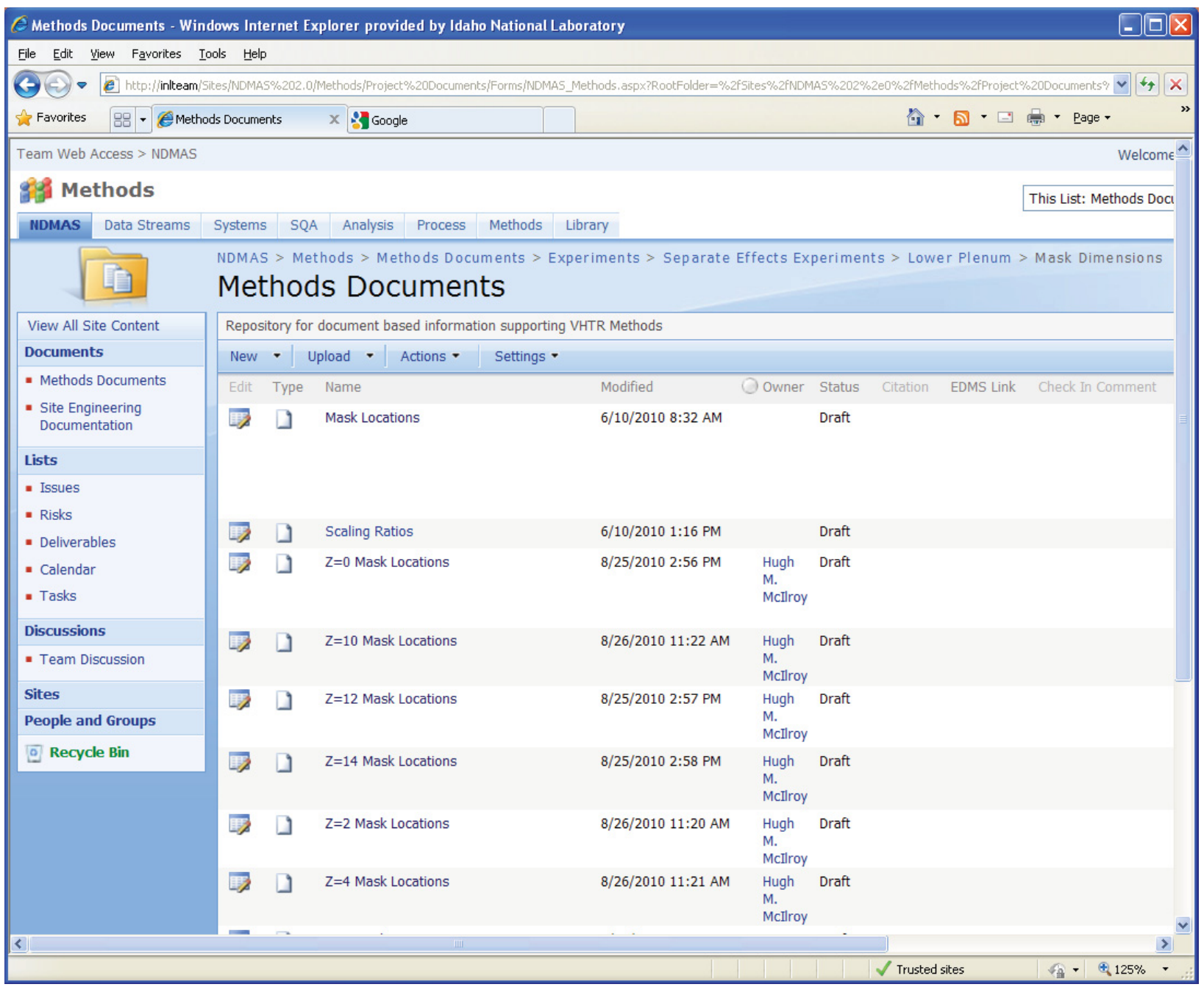

Figure 8. NDMAS SharePoint Lower Plenum AutoCAD drawings of non-fluid experimental regions used to mask particle image velocimetry (PIV) data.

\subsection{Analysis Explorer}

Analysis Explorer is a product developed by Phoenix Integration for use by computational scientists who need to keep track of hundreds of files associated with model configuration and execution. Analysis Explorer allows users to drag and drop directories and files associated with a model execution environment into an Analysis Explorer directory. The files move into an archive database and file metadata indexed to facilitate searching and version control. Analysis Explorer also provides a Web link for access from the NDMAS Web portal. This allows easy construction of a Web portal that provides searching, metadata, file access, and version control for models and simulation experiments.

Figure 9 shows the Analysis Explorer display of Lower Plenum archive data. The files show they are under version control and file B00005.dat shows currently checked out to user "alesrs." Viewing and searching of other information, such as notes, file properties, dependencies among files, and XML metadata is possible. The Analysis Explorer has an extensive application programming interface (API) used to change what information is stored as metadata or to automate processing of files dropped into folders in Analysis Explorer. 


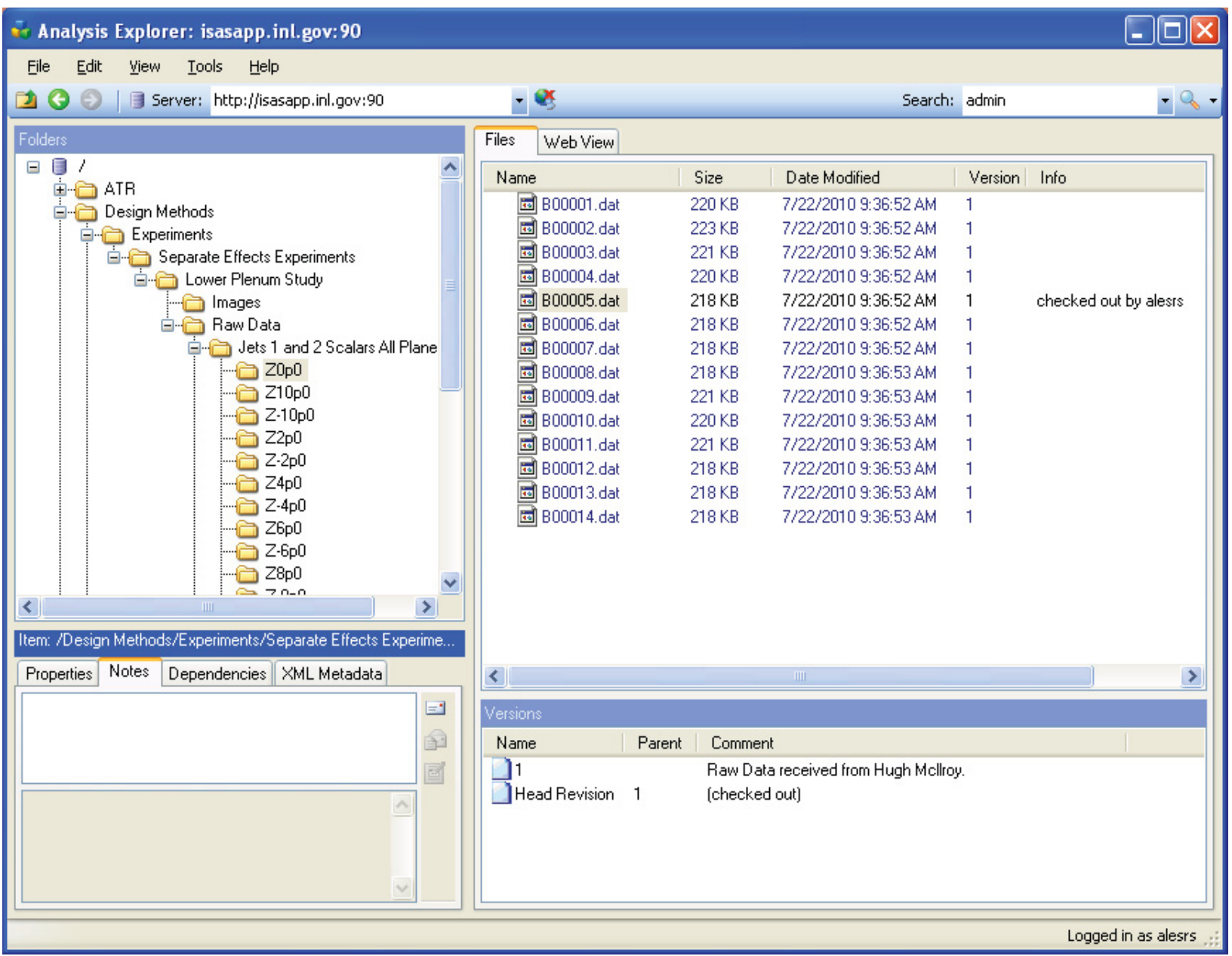

Figure 9. NDMAS Analysis Explorer displaying Lower Plenum study raw data files.

\subsection{SAS and JMP}

Users who have access to the network within the INL firewall can use SAS analysis tools and a companion product named JMP to access NDMAS information. SAS has an extensive library of statistical and modeling capabilities. The SAS analytical routines reside on a server accessed through client software installed on the user's computer. JMP, on the other hand, is a standalone program that resides on the user's computer. JMP has extensive exploratory data analysis and graphics capability in addition to special tools for design of experiments for simulation studies. JMP acts as a client to access SAS data libraries hosted on a central server in a manner similar to the previously described method for Excel.

\subsubsection{SAS}

SAS systems provide the main NDMAS Web delivery capability, but also provide extensive statistical analysis tools that are available to all Methods researchers. A more complete discussion of NDMAS's SAS capability can be found in (Gentillon et al. 2010) as well as on the internet at $\mathrm{http}: / /$ support.sas.com/resources.

SAS statistical users perform analysis by invoking a SAS procedure or PROC. PROCs greatly reduces the lines of code needed to perform numerical analysis. To demonstrate some of the capabilities 
of SAS, the remainder of this section discusses a procedure entitled PROC MODEL, which gives the user a sense of the analytical capabilities of SAS that are tightly integrated with NDMAS.

The PROC MODEL procedure ${ }^{1}$ is used to solve systems where relationships among variables represented by one or more nonlinear equations exist. Use PROC MODEL to estimate, simulate, and forecast nonlinear simultaneous equation models, Monte Carlo simulations, and goal-seeking simulations. Perform parameter estimation using the following methods:

- Ordinary Least Squares (OLS)

- $\quad$ Two-stage Least Squares (2SLS)

- Seemingly Unrelated Regression (SUR) and Iterative SUR

- Three-stage Least Squares (3SLS) and Iterative 3SLS

- Generalized Method of Moments (GMM)

- Simulated Method of Moments (SMM)

- Full Information Maximum Likelihood (FIML)

- General Log-Likelihood Maximization.

The next section presents an example of the use of PROC MODEL to solve a simple nonlinear circuit estimation problem.

\subsubsection{Nonlinear Circuit Estimation Example}

The following example of a PROC MODEL used for a nonlinear problem

(http://support.sas.com/documentation/cdl/en/etsug/60372/HTML/default/etsug_model_sect090.htm) shows a typical amount of code writing necessary to implement SAS procedures.

1. http://support.sas.com/documentation/cdl/en/etsug/63348/HTML/default/viewer.htm\#/documentation/cdl/en/

etsug/63348/HTML/default/etsug_model_sect001.htm 
Consider the nonlinear circuit shown in Figure 18.92.

Figure 18.92 Nonlinear Resistor Capacitor Circuit

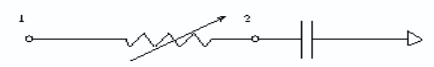

For the circuit in Figure 18.92, the relationships are

$C \frac{d V}{d t}=I$

for the capacitor and

$V=\left(R_{1}+R_{2}(1-\exp (-V))\right) I$

for the nonlinear resistor. The following differential equation describes the current at node 2 as a function of time and voltage for this circuit:

$C \frac{d V_{2}}{d t}-\frac{V_{1}-V_{2}}{R_{1}+R_{2}(1-\exp (-V))}=0$

This equation can be written in the form

$\frac{d V_{2}}{d t}=\frac{V_{1}-V_{2}}{\left(R_{1}+R_{2}(1-\exp (-V))\right) C}$

Consider the following data.

data circ;

input v2 v1 timede;

datalines;

$\begin{array}{llllllll}-0.00007 & 0.0 & 0.0000000001 & 0.00912 & 0.5 & 0.0000000002\end{array}$

$0.030911 .0 \quad 0.0000000003 \quad 0.064191 .5 \quad 0.0000000004$

$0.110192 .0 \quad 0.00000000050 .16398 \quad 2.5 \quad 0.0000000006$

$0.23048 \quad 3.0 \quad 0.0000000007 \quad 0.305293 .5 \quad 0.0000000008$

$0.39394 \quad 4.0 \quad 0.0000000009 \quad 0.49121 \quad 4.5 \quad 0.0000000010$ 


\subsubsection{JMP}

JMP is a SAS companion product generally used by individual analysts to perform statistical and exploratory data analysis. Figure 10 is a contour plot of the velocity profile from the Lower Plenum Jets 3 and 4 Experiment produced using JMP.

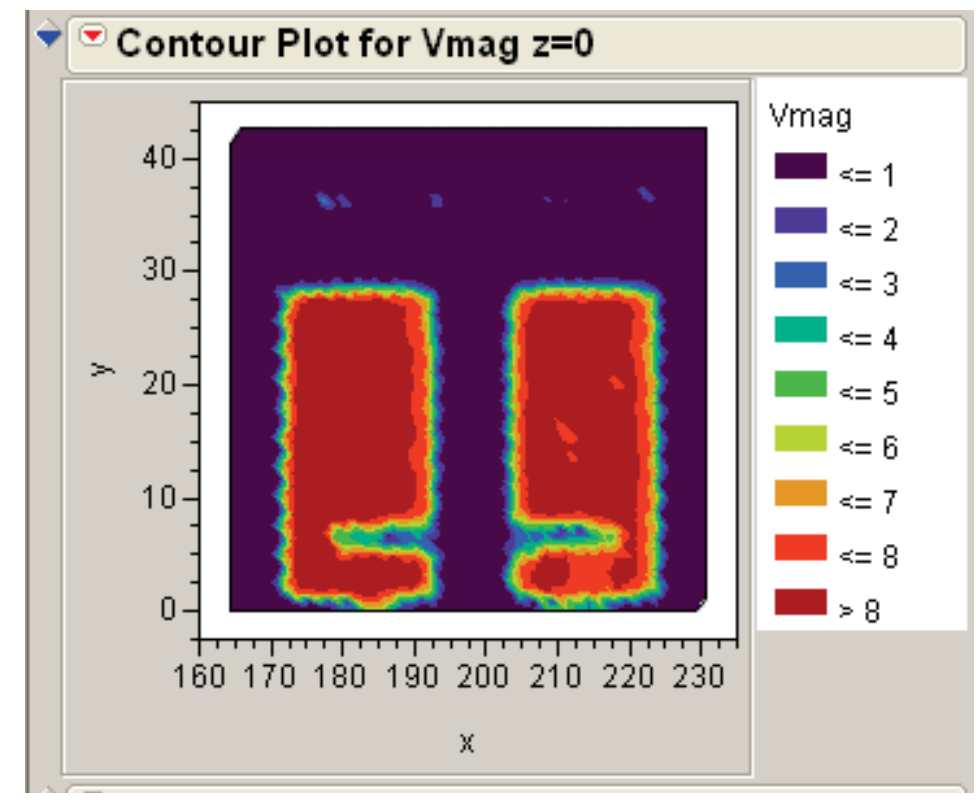

Figure 10. Contour plot created in JMP using Lower Plenum Jets 3 and 4 data.

\subsection{Other Modeling Tools}

Figure 11 shows the relationships among modeling tools used to achieve the analysis requirements of the Methods Program (Schultz et al. 2005). NDMAS has the capability to integrate these tools, using the ModelCenter interface, and the capability to read and write data directly to the NDMAS repository. ModelCenter (described in a later section of this report) can interconnect models, if appropriate, and conduct a whole system simulation run. 


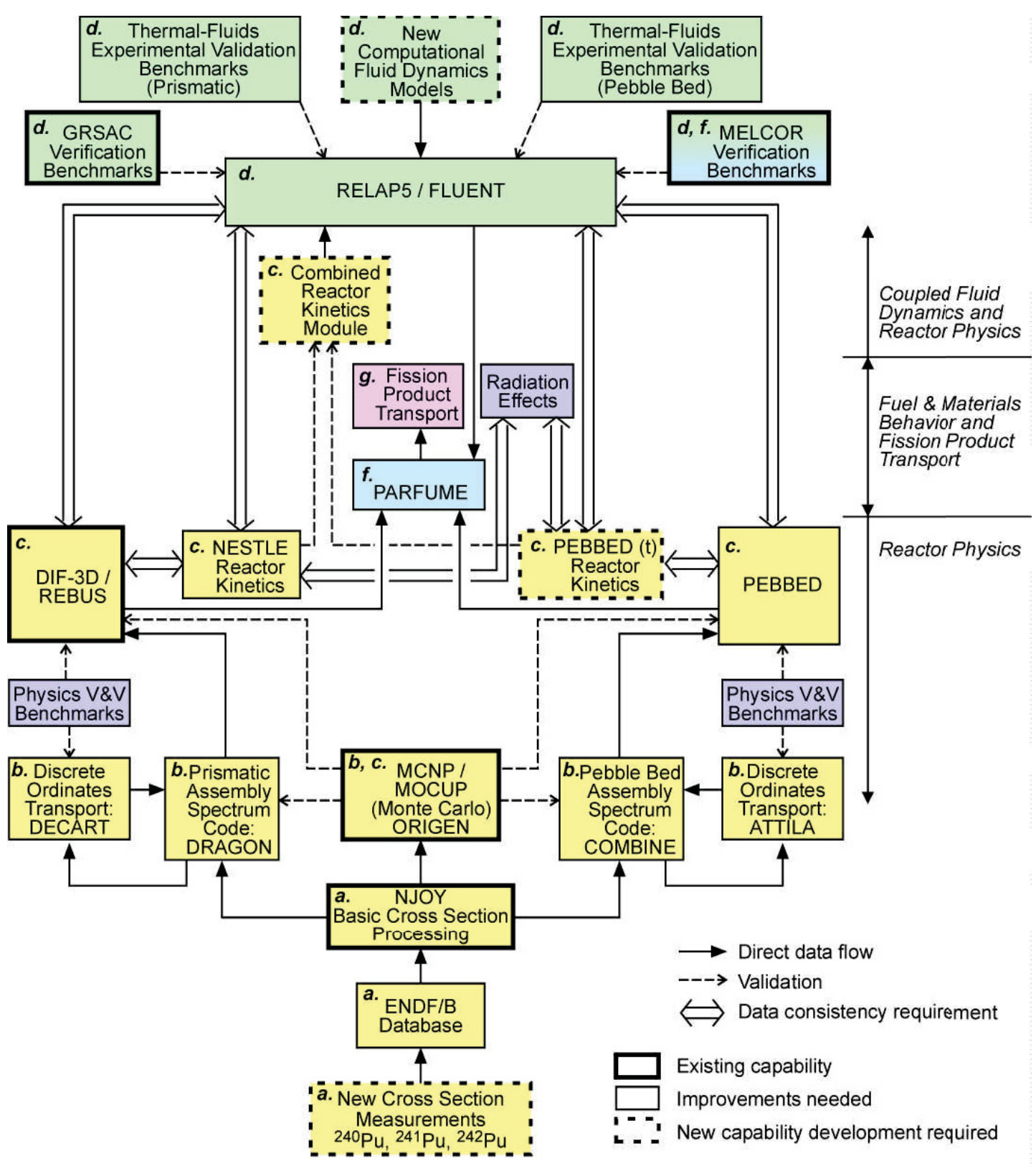

QA-GASก0B9-05

Figure 11. Software and models used to satisfy the analysis requirements of the Methods Program (Schultz et al. 2005). 


\section{EXPERIMENTAL DATA QUALIFIATION}

Experiments are conducted to produce data for testing the accuracy of very high temperature reactor (VHTR) simulation models. The NDMAS system receives the experimental data. PLN-2709 details the operation of the NDMAS data qualification process (Hull 2010).

\subsection{Lower Plenum Standard Problem}

The Lower Plenum Standard Problem is conducted in the Matched-Index-of-Refraction (MIR) facility located at INL. The purpose of these fluid dynamics experiments in the MIR flow system at INL is to develop benchmark databases for the assessment of computational fluid dynamics (CFD) solutions of the momentum equations, scalar mixing, and turbulence models for typical VHTR plenum geometries in the limiting case of negligible buoyancy and constant fluid properties.

The objectives of the experimental program are to (1) build numerical calculation models of the important VHTR thermal fluids phenomena already identified, and (2) demonstrate that the models have sufficient capability to calculate the important phenomena within a prescribed acceptance criteria by using high fidelity validation data. These computational capabilities need validation by comparison to experimental and analytical benchmark data. In turn, management of these data sets requires implementation of appropriate statistical and quality assurance procedures.

To achieve these experimental objectives, INL has developed a large MIR flow system that uses optical techniques, such as laser Doppler velocimetry and PIV, to obtain flow field measurements in complex passages without disturbing the flow. Figure 12 shows the experimental apparatus. Matching the refractive indices of the fluid and the physical model ensures that there is no optical distortion. The large size provides good spatial and temporal resolution and provides a means to measure flow fields, turbulence, and mixing in the complex geometry of a prismatic VHTR lower plenum. Usage of the resulting data validates CFD codes and their turbulence models for the limiting case of dominant forced convection where temperature is a passive scalar.

The NDMAS also stores the experimental geometry associated with these experiments into the data

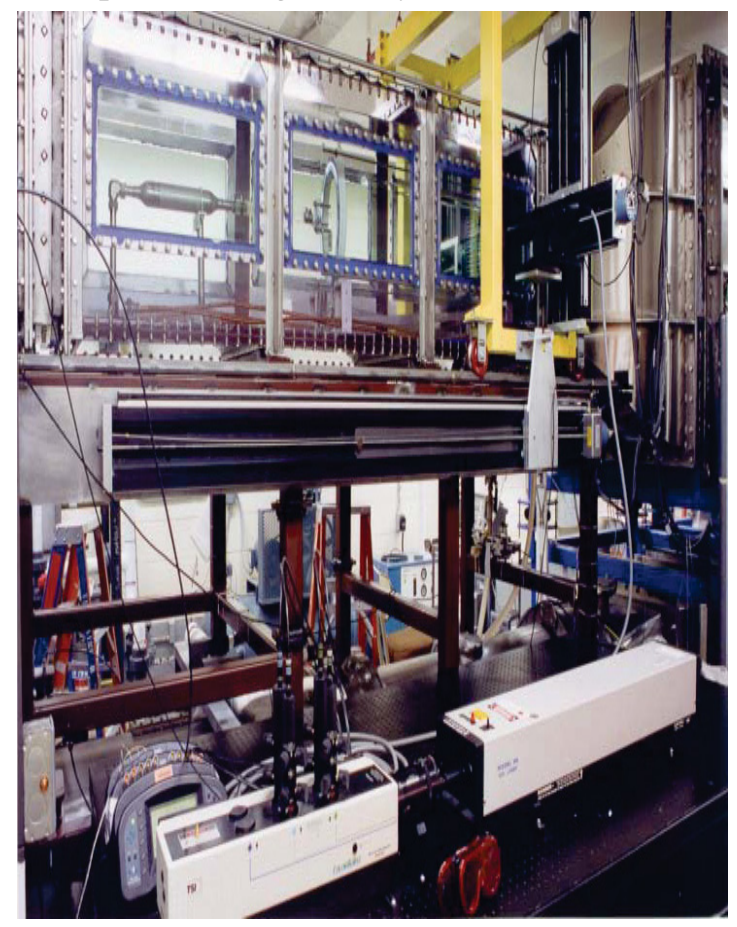

Figure 12. INL MIR facility. 


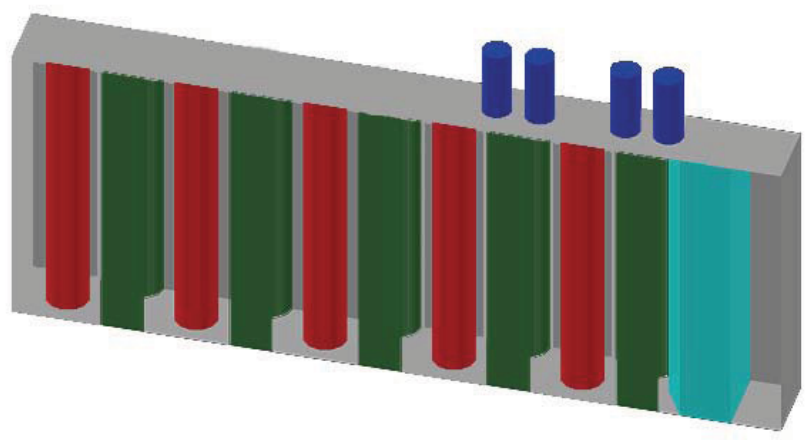

Figure 13. Drawing of the scaled model used in the INL MIR facility to obtain flow data. Flow enters in from the top inlets and flows out toward the left rear; cylinders represent core support columns.

management and acquisition structure. Figure 13 shows a model of the flow cell placed within the flow field established in the INL MIR facility. The resolution required by the MIR experiments to develop understanding of the magnitudes of experimental uncertainties from the results obtained requires special data acquisition software and results in large data sets. A 3-D PIV system from LaVision, Inc. obtained velocity field measurements. DaVis 7.1 software controls the system. DaVis is a charged coupled device image acquisition program developed by LaVision and controlled by a LaVision dual-processor programmable timing unit.

Data management and analysis plays a major role in requirements for use of the experimental results. Criteria considered when using MIR data as a benchmark database include:

- Objectives of the database and its experiments

- Precise details of geometries and materials employed (as built dimensions)

- Details of the instrumentation and measuring/sampling techniques

- Sizes of measuring control volumes, sensors and probes, light sheet thicknesses, etc.

- Inlet flow conditions, including turbulence distributions, heat loss calibrations, etc.

- Determination of desired fields in the region(s) of interest

- Tabular presentation of the data

- Analyses of the experimental uncertainties of all quantities involved

- Electronic storage of information in a format useful to CFD analysts in multiple forms

- Appropriate graphical representation of key results

- Careful, readable documentation of details

- Comparison to calculations before release

- Web accessible and controlled data

- Long-term point-of-contact.

For proper benchmark databases, the experimental uncertainties of all the measured quantities and their propagation into the results must be estimated quantitatively. In some cases, mean statistics will be adequate, in others, instantaneous values will be desirable. In either case, knowledge of uncertainties in the instantaneous quantities will be necessary to perform a reasonable uncertainty analysis. 


\subsection{Lower Plenum Portal}

Data from the Lower Plenum Standard problem is available within the "Design Methods" Web portal (http://isasapp.inel.gov/SASPortal). Figure 14 shows the main Lower Plenum portal. A brief description of the experiment links to background information, and diagrams of the process and geometry of the experiment (not shown) are displayed. The left panel indicates nine sub-portlets that present tables and graphs of the composite and individual data sets.

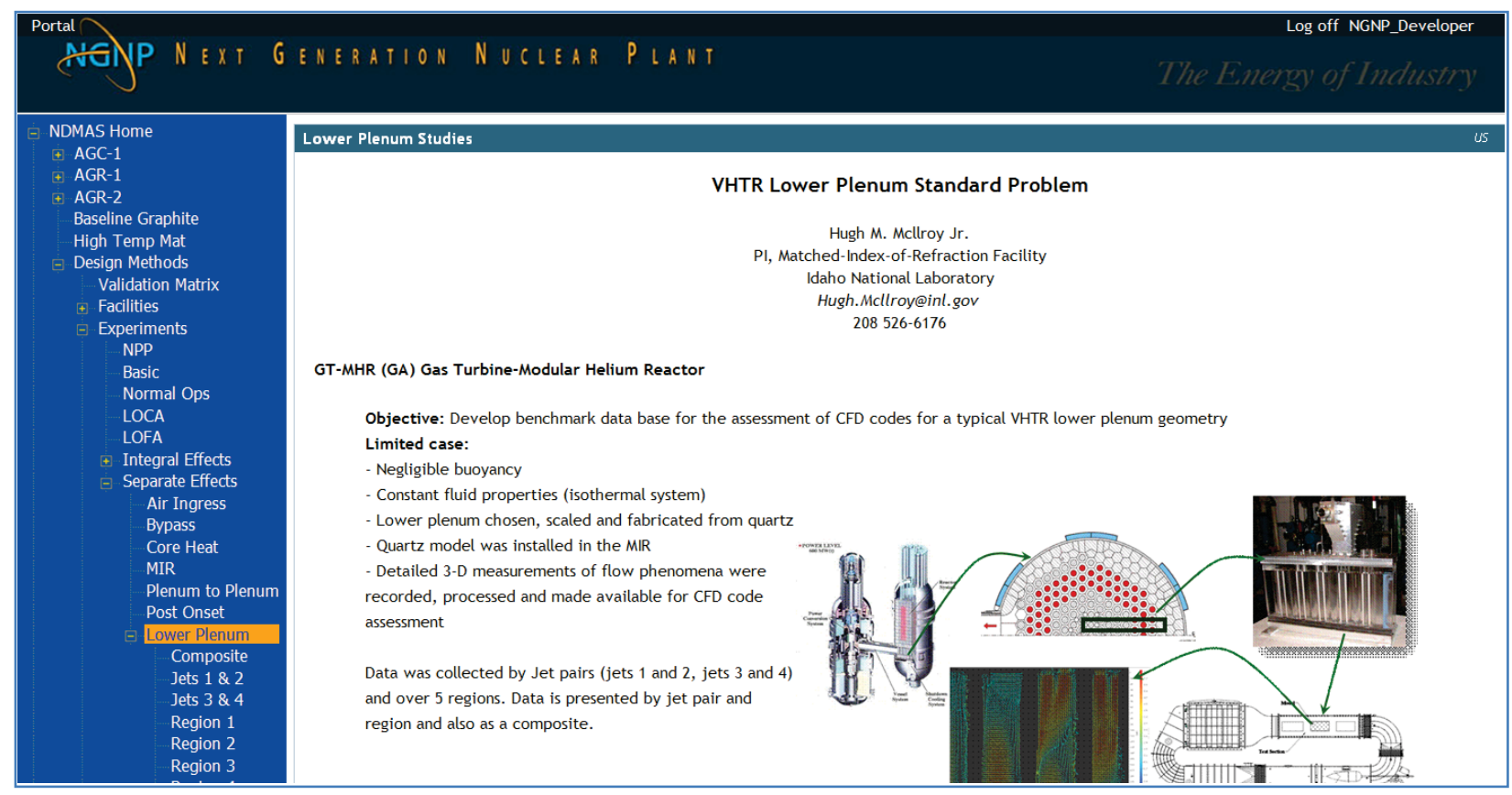

Figure 14. NDMAS Lower Plenum standard problem portal.

Figure 15 shows the composite portal made up of three portlets: the top portlet describes the project; the middle includes the SAS report discussed next; and the third displays flow vectors for a composite of all study data. Scrolling provides access to 23 graphs, one for each scan plane (like the one shown). Vector length and color indicate velocity magnitude. Hovering the mouse pointer over an individual vector will open a data grid that displays additional data associated with the vector. Plans also include contour graphs and 3-D flow graphs.

Once reviewed, this portal will be available on the NDMAS external Web portal for viewing by DOE Headquarters and other customers. 


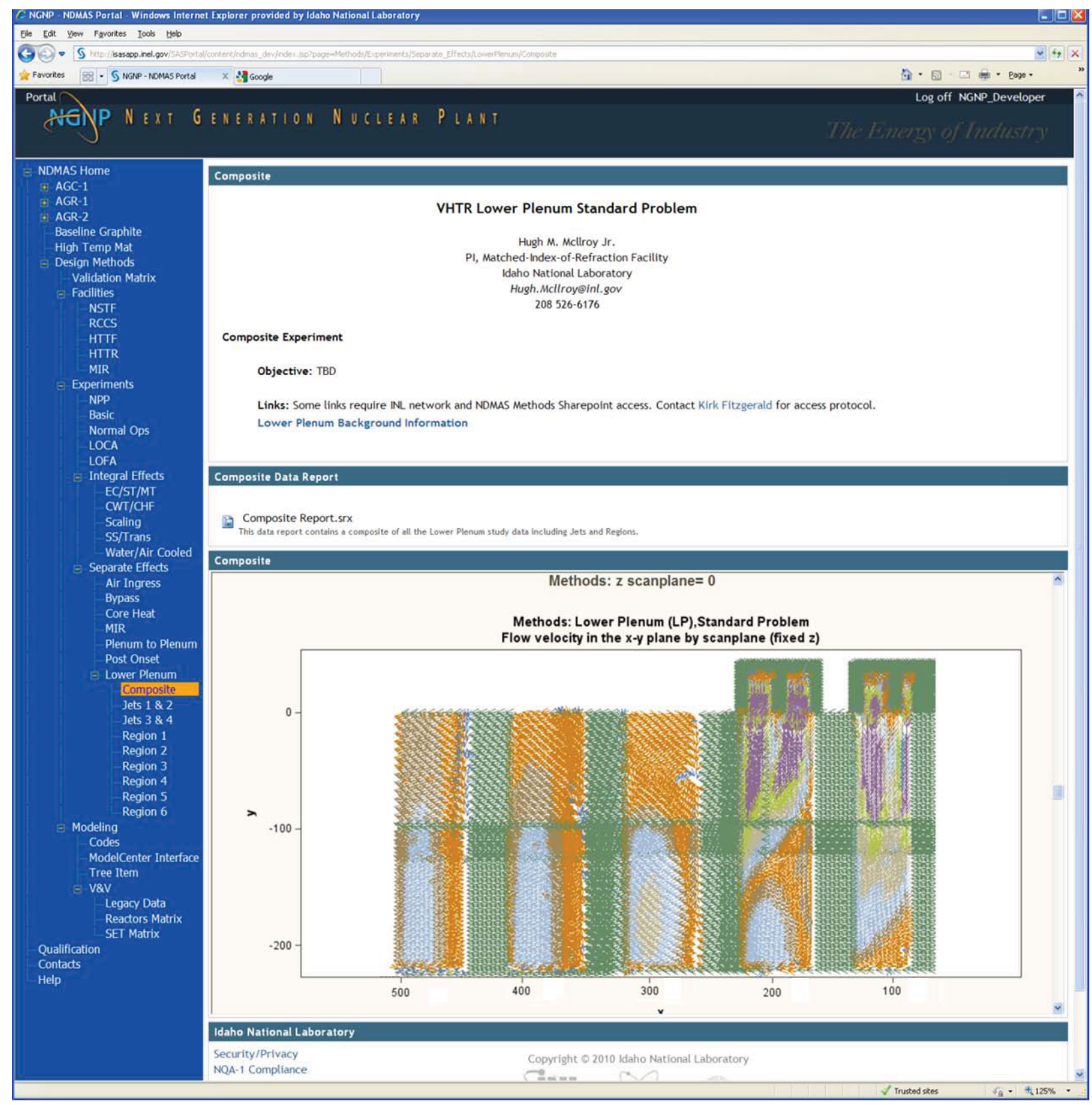

Figure 15. Lower Plenum portal for the composite of all the experimental data.

Figure 16 shows the result of clicking on "Composite Report.srx" located in the middle portlet. Shown is a drillable table, followed by graphs of the data. The blue + and down arrow in the column labeled ' $z$ ' can be used to expand and drill into the data by expanding to the ' $x$ ' and then ' $y$ ' values. Values presented are averages unless the user has drilled to a particular $\mathrm{z}, \mathrm{x}$, or $\mathrm{y}$ location to display the measured data value. The data grid linked to the graphs enables drilling into the data and changes the data presented within the graphs.

Users can edit this report to change the variables displayed, include other graphs, filter the data, apply highlighting conditional on data values, and add new data columns that are algebraic combinations of existing data columns. The displayed data and/or graph data is downloadable into Excel on the user's computer. 


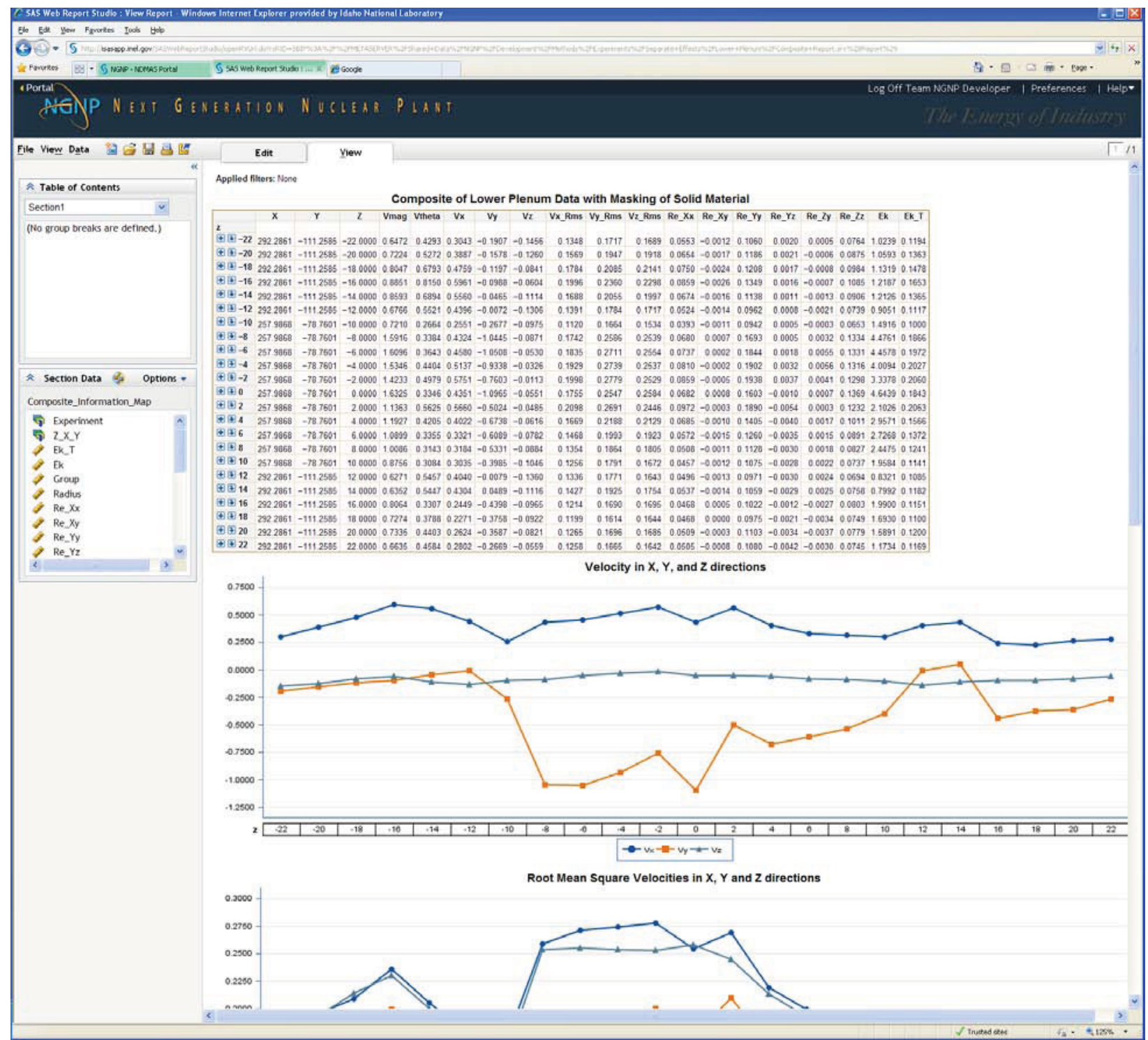

Figure 16. NDMAS report of composite Lower Plenum data.

Figure 17 shows how the graphs have been rescaled result from a drilling of data at $\mathrm{z}=-4 \mathrm{~mm}$ and $\mathrm{x}=$ $91.01 \mathrm{~mm}$ ( $\mathrm{y}=0$ is to the right, increasing negative to the left). Displayed are velocity, RMS velocity, and Reynolds stresses variables. Other graphs (not shown) display kinetic energy. 


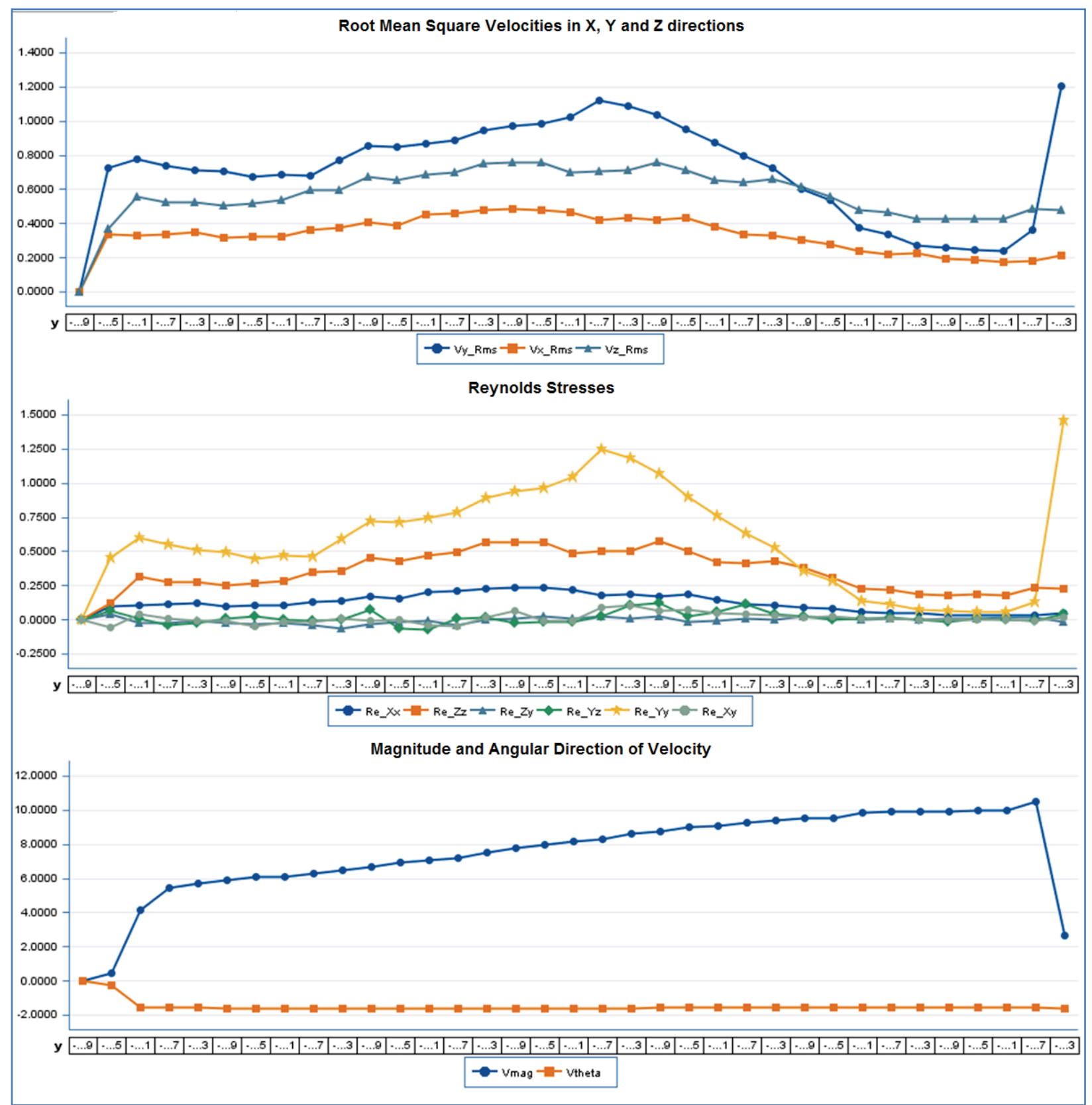

Figure 17. Scalable graphs of Lower Plenum data along a vertical profile (y axis) for $\mathrm{z}=-4 \mathrm{~mm}$ and $\mathrm{x}=$ $91.01 \mathrm{~mm}$.

An additional feature of these SAS reports is that users can customize them to their individual needs and download the information into Excel, Word, or PowerPoint.

Not shown are portals for each of the sub experiments that currently have the same information for a subset of the data. 


\subsection{Lower Plenum Data Qualification}

Quality control tests of the Lower Plenum data checked for duplicates and missing values. Project personnel can request additional quality tests. Figure 18 shows the NDMAS environment for qualification testing. Production of a variety of reports and alerts document any data exceptions found. These reports and alerts provide information on the provenance of data within NDMAS.

The Lower Plenum data contained no duplicate or missing data.

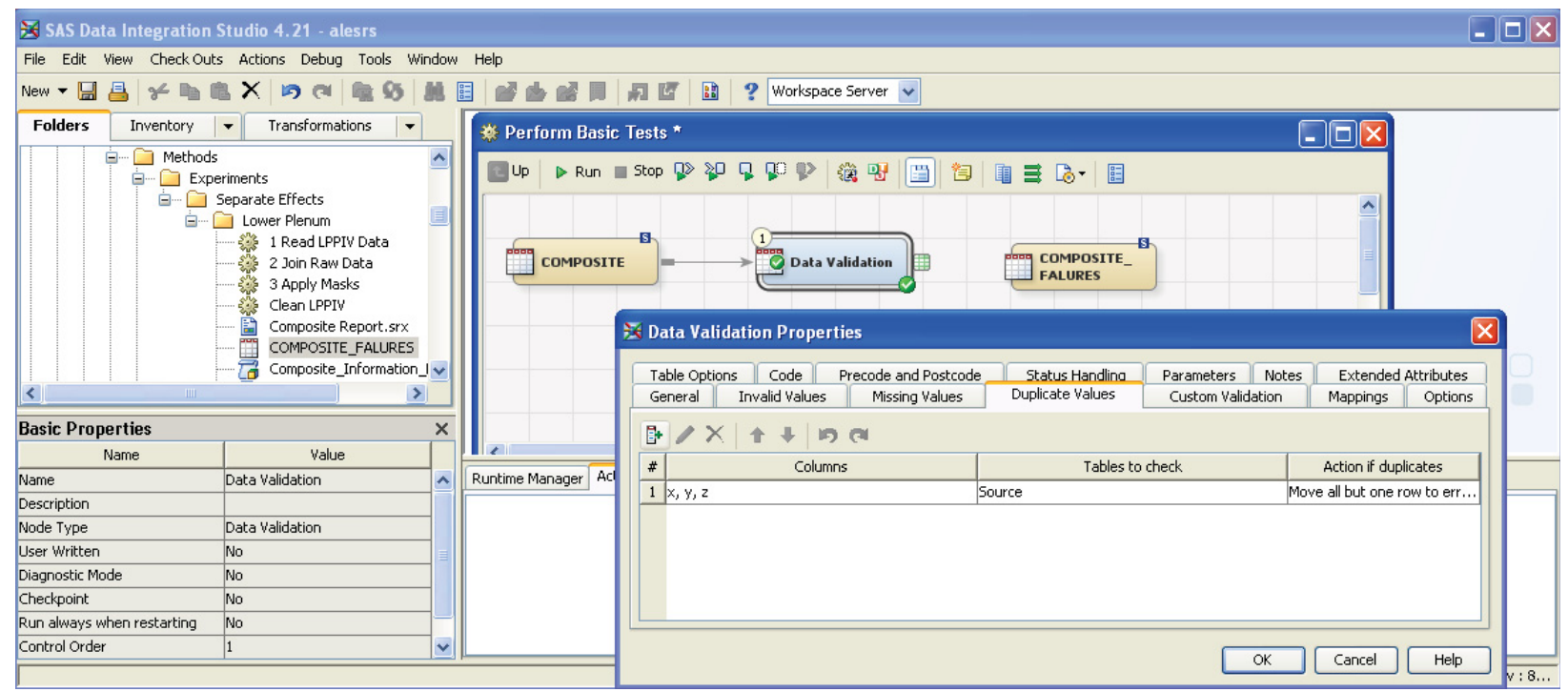

Figure 18. NDMAS data validation and qualification environment. Dialog shows point-and-click capability to test data for invalid values, missing values, duplication, and custom tests. 


\section{MODEL DATA QUALIFICATION 4.1 INTRODUCTION}

NDMAS captures data generated by NGNP models that perform simulations of reactor components and systems. Data derived from models complicates the maintenance of data provenance, since provenance is degraded when data is downloaded from a controlled database to personal computers (Excel, text, binary, etc.), fed as input into models, generated as results (Excel, text, binary, etc.), and then uploaded back into a database repository. Provenance in such an environment, if done at all, requires time-consuming hand entry methods.

The Methods group of the NDMAS project is developing tools that will allow these scientists and engineers to submit their simulations and analyses to a database. This facilitates the publishing of results to a Web portal where DOE Office of Nuclear Energy, NRC, and other interested parties can read them. The quantity of data produced would be prohibitive to capture except by individuals taking responsibility to submit their own work to an NDMAS database. Tools are therefore needed that will make it easy to collaborate, share, and control the simulations and data created in designing the next generation reactors to meet NQA-1 requirements.

Developing processes, procedures, and tools for the engineers to submit their work to an archive or central database started with the purchase of several commercial applications. A SAS application suite provides database support, statistical analysis, and part of the Web portal infrastructure. Tools from Phoenix Integration called ModelCenter and Analysis Server are used to automate analysis processes, irrespective of what computational platform is being used. Analysis Explorer provides tools to create an analysis library to preserve provenance of data and codes used during model runs.

To improve provenance of data used and generated by simulation codes, NDMAS has developed the capability to execute models linked directly to the NDMAS data repository-both to receive input parameterization and to store output of simulated results. This means that data does not need to transfer from (e.g., from db to Excel) or be manually stored at a location (server to local computer) prior to being input or output from a model. This approach reduces data errors and improves data provenance and, thus, data quality. Analysts and auditors benefit from information associated with the model runs that require preservation.

Though maintaining data provenance for Methods model runs is important, it must not become a burden for computational scientists. Figure 19 shows how NDMAS can provide an interface to an individual Methods researcher's work environment without affecting their currently preferred approach to performing their research.

In the Figure 19 example, the computational scientist is running a program named Origen2. The file directory shown lists all the files needed to support a model run. The model is executed by starting one of the two executable files (O2_FAST.EXE or O2_THERM.EXE) stored in the directory. These programs expect to find input and output files in specific directories.

ModelCenter adds a ".template" file (see red arrow), also called a wrapper, to the directory. This file tells ModelCenter how to read and write inputs and outputs and run the Origen2 executable. This file is used in ModelCenter by simply dragging the icon shown by the arrow into the main ModelCenter workspace. Model parameters can then be changed within ModelCenter, the model can be executed, and the results returned to ModelCenter for review. The two other icons appearing in the ModelCenter workspace transfer data to and from the NDMAS repository. 


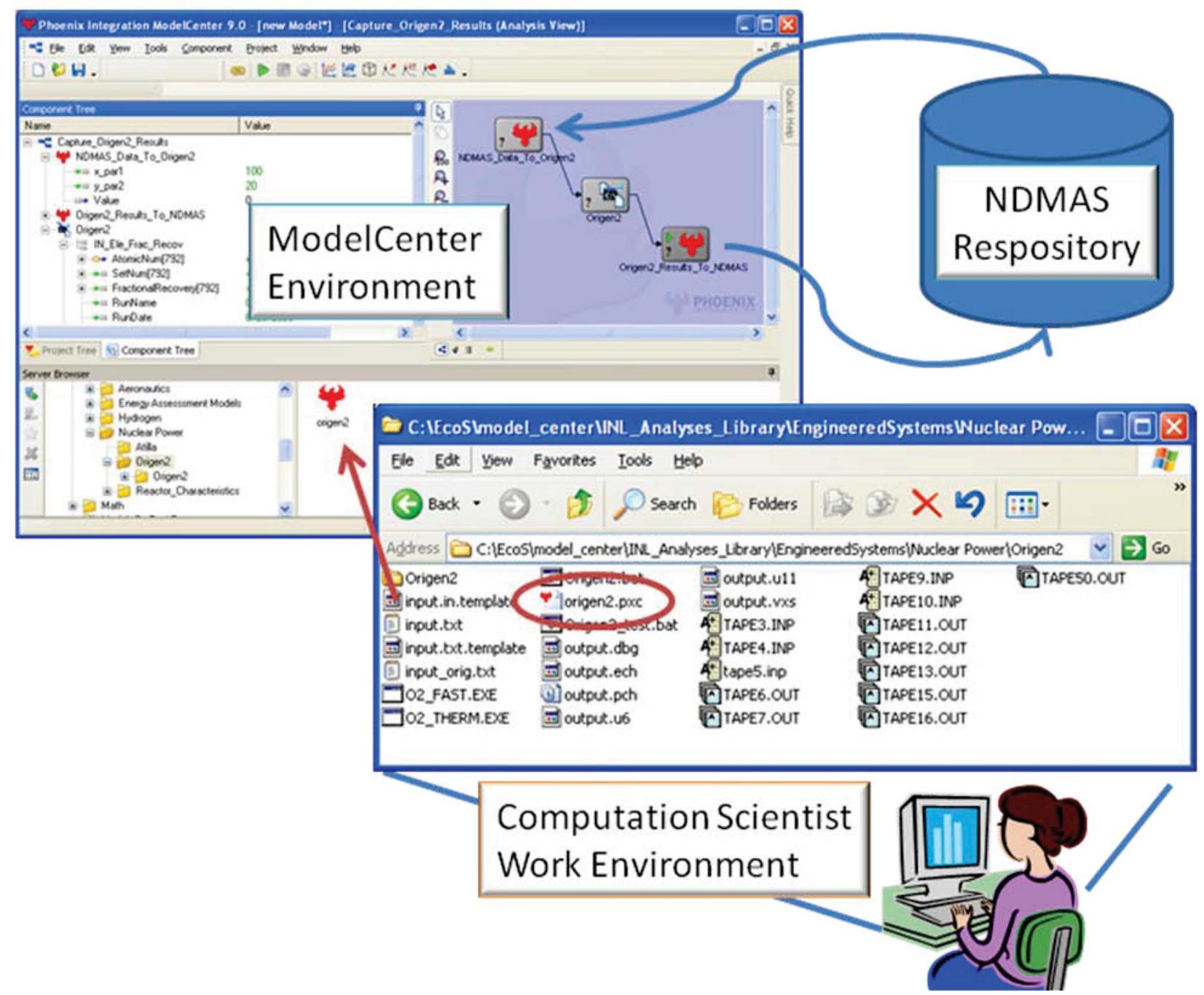

Figure 19. Cartoon showing the relationship between an individual computational scientist's work environment and ModelCenter data transfer to the NDMAS repository.

In this way, ModelCenter is nonintrusive to the normal work patterns chosen by the computational scientist. Model configuration files can be manipulated in the normal manner, and when it becomes important to preserve a model run, the user simply opens ModelCenter and runs the Origen2.pxc model (circled in red in the file directory) transferring data to NDMAS. ModelCenter is expected to only be used for model runs where there is either an interest in preserving the model run configuration (perhaps associated with a final analysis report) or when it is desirable for model output data to be delivered to the Web environment for access by other researchers or customers. NDMAS's goal is not to burden researchers with extra tasks, but to automate to the extent possible, data quality and provenance tasks associated with model runs.

The main requirements for Methods model integration are:

- Provide means to transfer data among interacting models and the NDMAS data repository

- Store relational information about model input and output data on a model runtime basis

- Capture and store the model operating environment, run state and version information as meta data for simulation result provenance 
- Provide a statistical analysis environment to support model verification and validation $(\mathrm{V} \& \mathrm{~V})$ and uncertainty analysis associated with experimental and model data

- Provide a Web-based delivery platform for model results and model V\&V, and uncertainty analysis.

This section will give an overview of the model integration environment chosen (ModelCenter) and will explain the integration of models within the NDMAS data repository.

\subsection{MODELCENTER ENVIRONMENT}

Methods model integration selected ModelCenter, a commercial model integration environment, to support Methods model integration. ModelCenter is a visual environment for software integration and model execution as shown in Figure 20. Using ModelCenter, Methods researchers can graphically create a system or process model by dragging and dropping applications from one or more servers (e.g., analysis servers) that provide an interface to external models such as MatLab, Fluent, Star CCM+ and virtually any other modeling environment of interest that has an API.

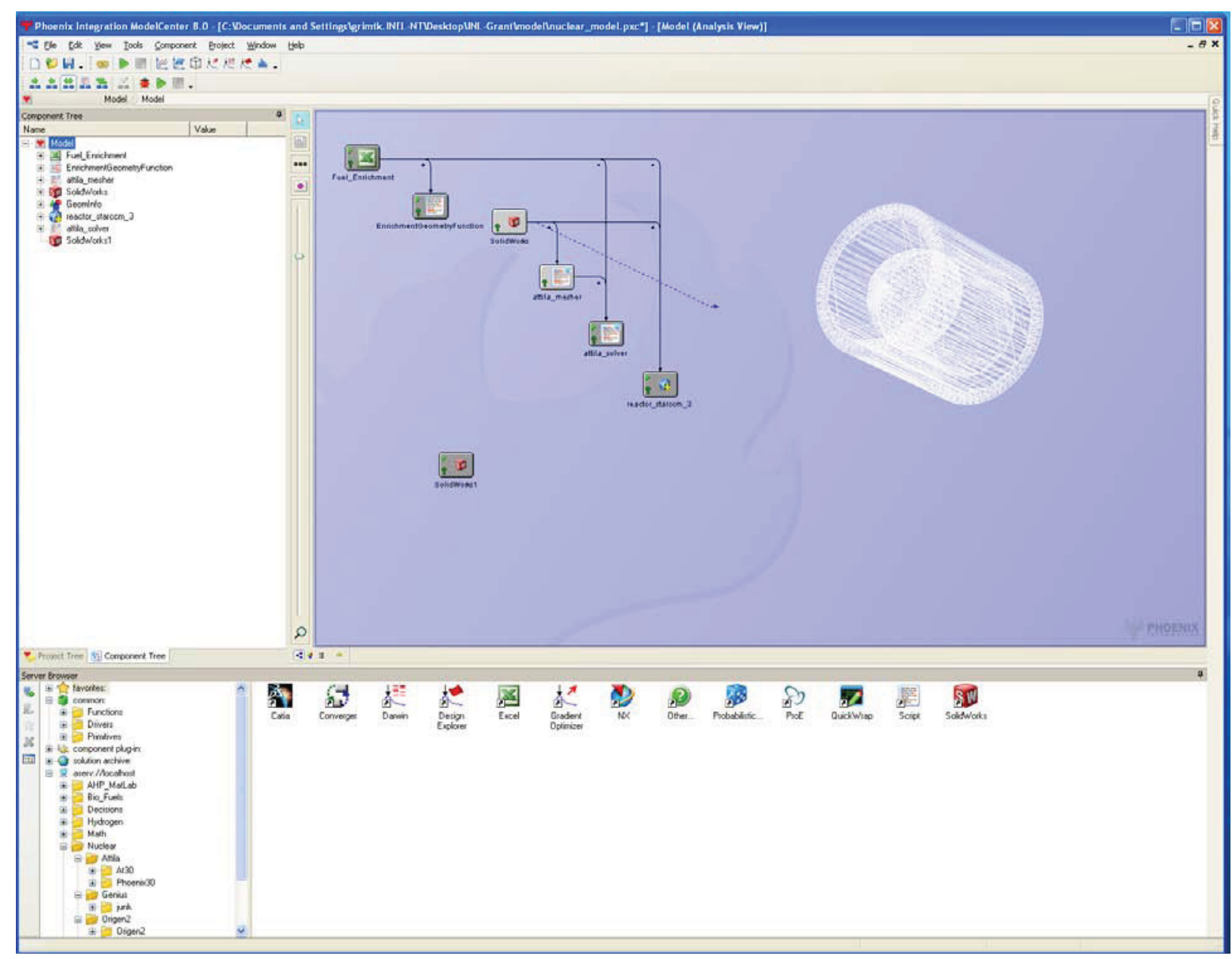

Figure 20. ModelCenter interface showing space nuclear integrated design model using Excel, a FEM mesher, Star CCM+, and Attila.

The graphical Link Editor shown in Figure 21 allows engineers to specify data passed from one application to the next when the model runs. The resulting integrated model may contain applications that reside on a number of different computers and execute on different operating systems. 


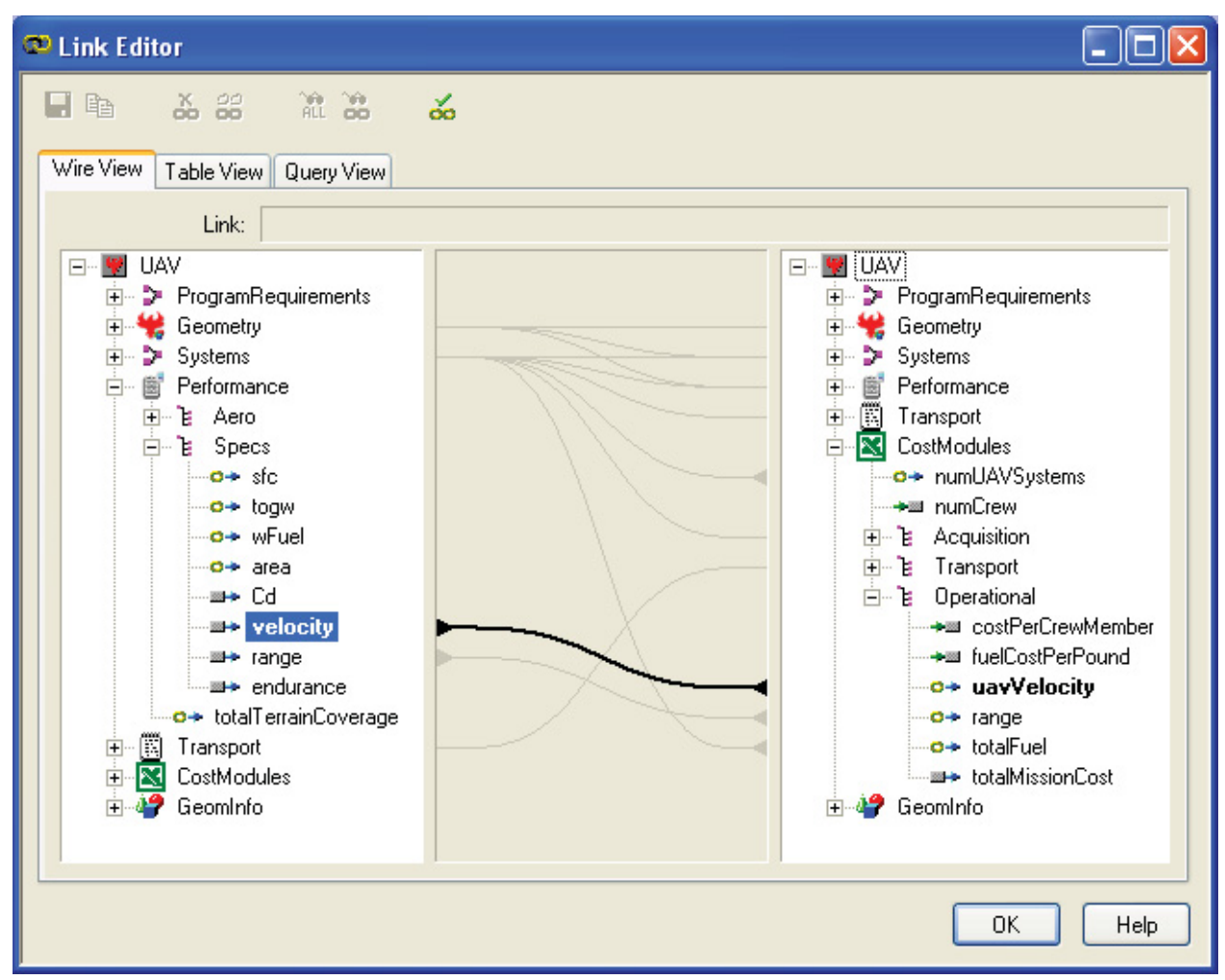

Figure 21. ModelCenter's Link Editor allows engineers to specify data passed from one application to the next when the model runs.

Once the model is created, users can automate the execution of the model and perform a wide range of trade-studies (parametric studies, design of experiments, optimization, Monte-Carlo analysis, etc.). Built-in visualization tools can be used to interpret and analyze the results, and the data can be exported to external applications. ModelCenter's open architecture allows users to seamlessly integrate their own (or third party) trade-study, optimization, and visualization tools into the environment desired.

\subsection{NDMAS MODEL INTERFACE}

ModelCenter is a tool used to enhance workflow automation using a graphical environment for process integration. It runs in a client/server mode, ModelCenter being the client and Analysis Server being the server. ModelCenter only runs on a Windows platform, but by collaborating with Analysis Center, applications on Linux platforms can be included in ModelCenter models. Linkage of different analysis codes through their inputs and outputs in a logical fashion using the ModelCenter concept of plug-ins is possible. Customization of ModelCenter for an organization's applications by writing plug-ins is possible. Any application can be "wrapped" (in other words, have a plug-in created) if it has a command line interface. Strictly graphical user interface programs may or may not be able to be wrapped, depending on whether an API is available.

An example ModelCenter model might connect a text file, an Excel spreadsheet, and an Abaqus run. Data in a text file can be read by ModelCenter and passed to an Excel spreadsheet that computes a value needed in an Abaqus simulation. ModelCenter retrieves the value from the spreadsheet and passes it to Abaqus. The Excel spreadsheet and ModelCenter would be running on a Windows machine and Abaqus could be running on a Linux-based high performance computing machine. Figure 22 shows Analysis Center running with two connections to applications running on a local machine. Connections to other machines could also be present. 


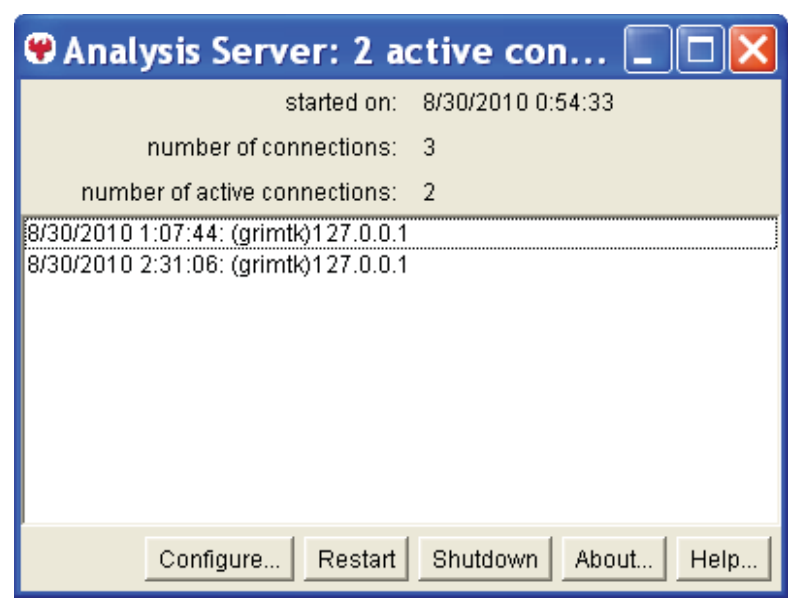

Figure 22. Analysis server running on a Windows platform.

The NDMAS project is using ModelCenter as a means for engineers to easily add analysis results to the NDMAS Web portal and possibly to submit their work to the NDMAS repository, thereby ensuring data provenance tracking. Current work focuses on developing the ability to call ModelCenter from the hierarchical Web portal. (Figure 23 is a picture of the Web portal.) This involves developing a .jsp (Java Server Page) script that is called from the portal that in turn calls the ModelCenter API.

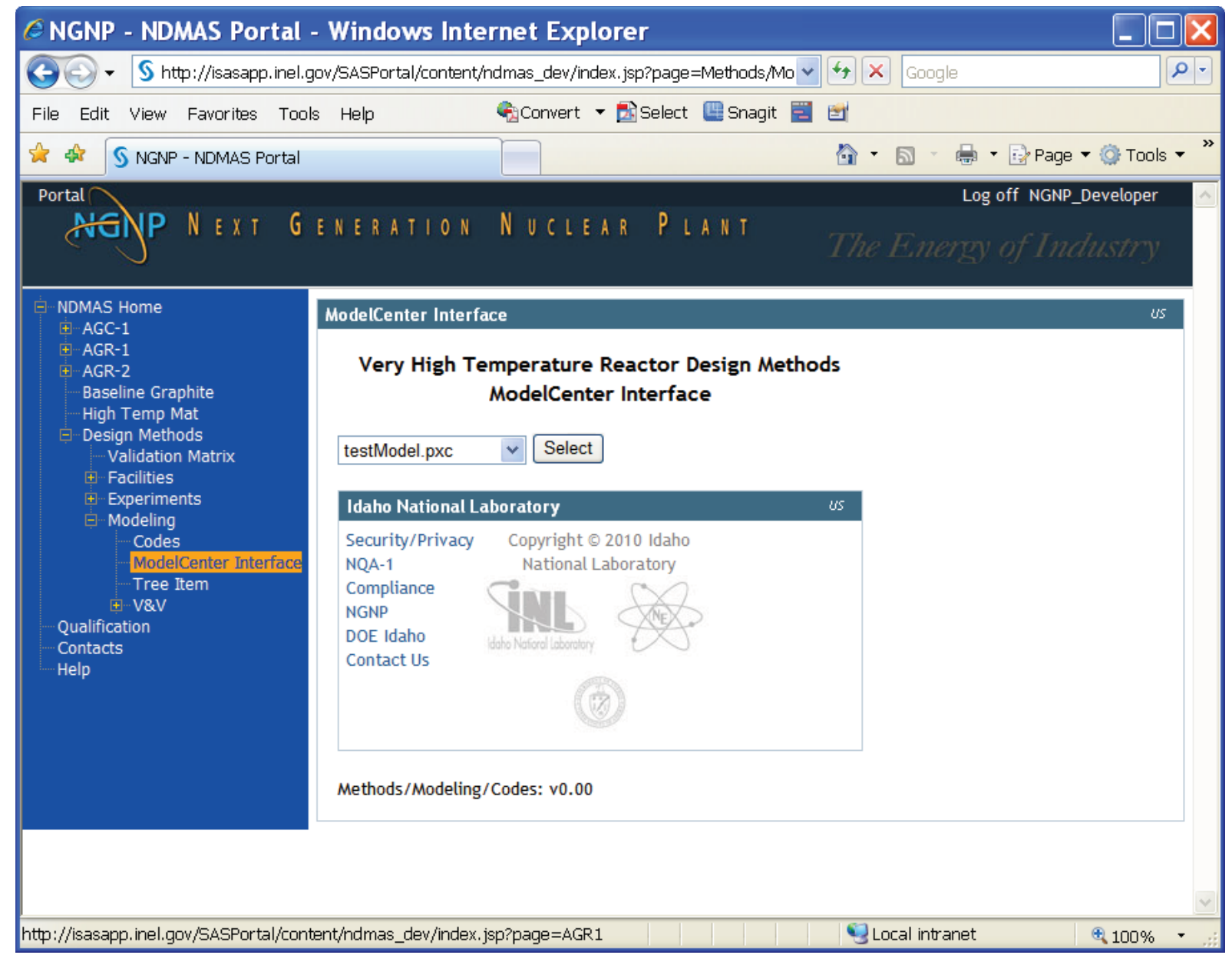

Figure 23. NGNP Web portal. 
This allows for server side (meaning the Web server machine versus the user's machine) running of ModelCenter, thereby providing for easier system maintenance and security. However, this objective is being approached in a systematic fashion to learn how to work with ModelCenter's API.

Figure 24 shows an example of a webpage developed as a first step in learning how to call ModelCenter from a webpage. A Matlab-based thermocouple prediction model was called. The small window shows Matlab running.

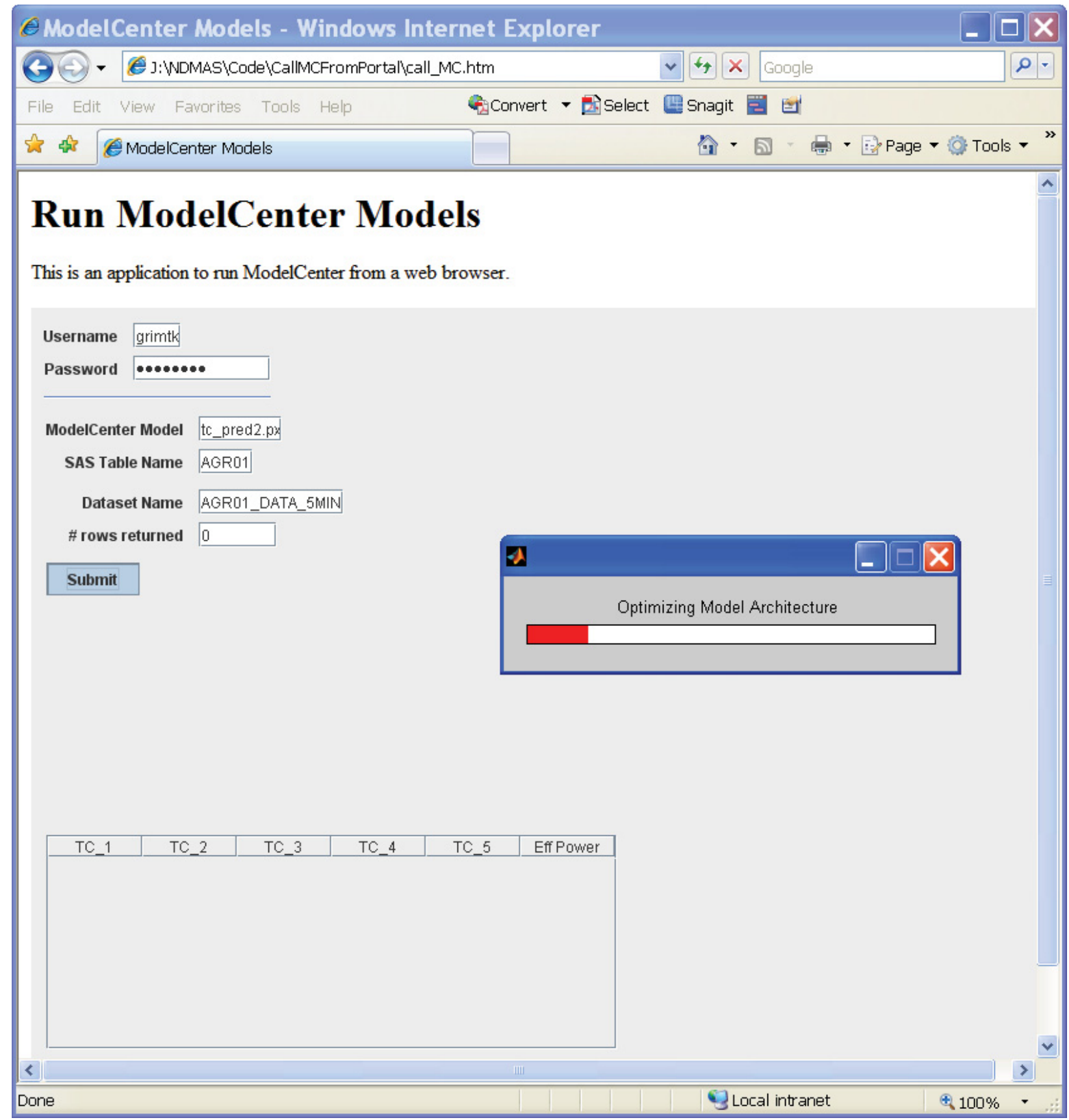

Figure 24. Sample webpage that calls a ModelCenter model that runs a Matlab-based thermocouple prediction model and displays back the results. 
Our prototyping of this approach has informed NDMAS 2.0 design decisions about how best to display the data returned from ModelCenter, how to store the ModelCenter models (as SAS stored procedures or as stand-alone files in the file system), and how ModelCenter can be used to aid the capture of simulation models and results.

Other work has centered on developing a proof-of-concept plugins for ModelCenter that allows the reading of data from SAS and storing results back to SAS. This plugin was developed at INL and is called the ModelCenter/DataPipe (e.g., ModelCenterDbPlugin shown in Figure 25).

To demonstrate the approach, a ModelCenter model was developed that reads Advanced Test Reactor thermocouple data from SAS into ModelCenter (Figure 25). The data is passed to a ModelCenter Matlab plug-in that runs a Matlab procedure, which predicts values for missing thermocouple data. The results are then written back to SAS/NDMAS.

The plug-in in the upper-left corner of the display area is a scripted plug-in developed to read data from SAS and import it into ModelCenter. The plug-in in the lower right is another scripted code that writes the results from the Matlab analysis back to a SAS table. The middle plug-in is a Matlab plug-in included with ModelCenter. It provides an editor in which the Matlab procedure can be entered as shown in Figure 26.

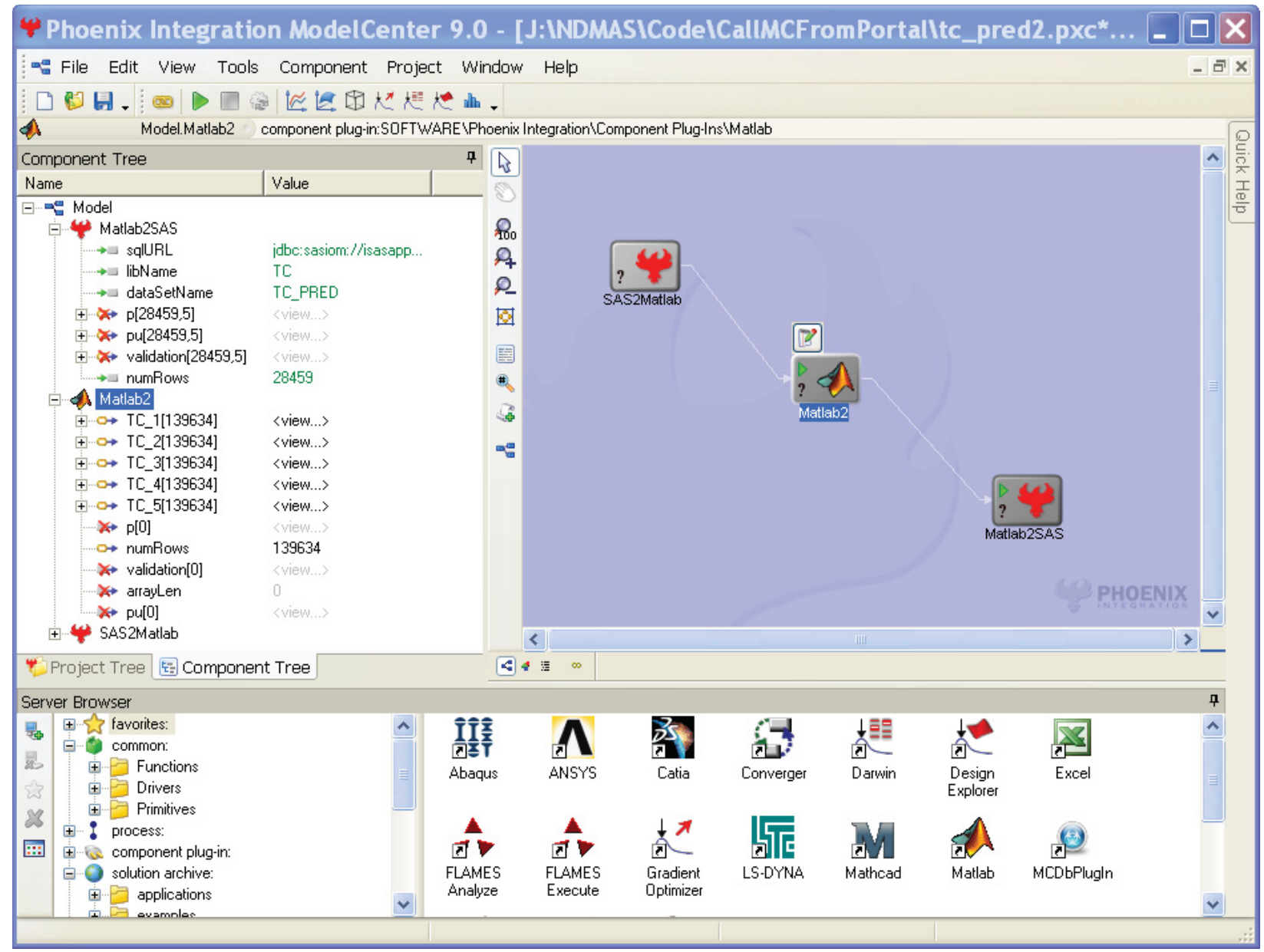

Figure 25. The ModelCenter thermocouple prediction model. 


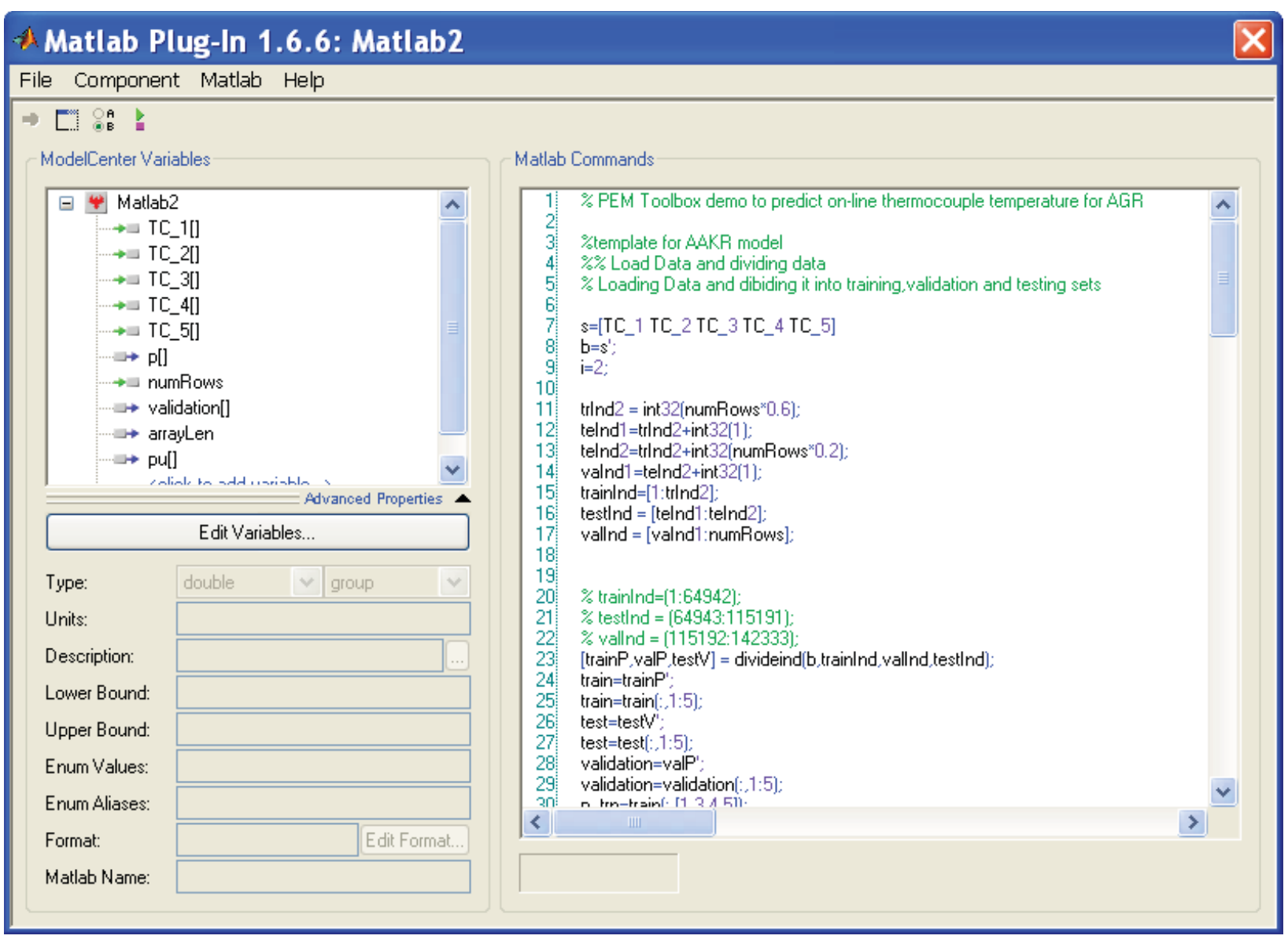

Figure 26. ModelCenter Matlab-plugin editor.

Plots and other output generated by Matlab are viewable after the Matlab process is run and, when saved to disk, can be viewed using the NDMAS Web portal without having to run Matlab directly. This can save the analyst time, since the NDMAS Web browser can simply be refreshed to display new results from the Matlab run and deliver it to other users by simply changing user permissions.

Analysts can use ModelCenter and SAS's API to construct scripts to link models ${ }^{\mathrm{m}}$ or they can use the special ModelCenter/DataPipe plugin shown in Figure 27 to construct Structured Query Language (SQL) to access NDMAS data using a point-and-click interface. The DataPipe provides a means for results of an analysis to be both read from and written back to NDMAS. The DataPipe manages to obtain the user's credentials from the system, allowing a single sign-on experience for the user. The end-result of using the DataPipe is to allow analysts to develop ModelCenter models without having to develop their own application-specific scripted plug-ins with hard-wired SQL queries. Figure 27 shows this DataPipe interface with a query used in the thermocouple prediction model to get ATR thermocouple data.

$\mathrm{m}$. A copy of the script code used to create the plug-in that reads data from SAS for the thermocouple prediction model is included in the appendix. 


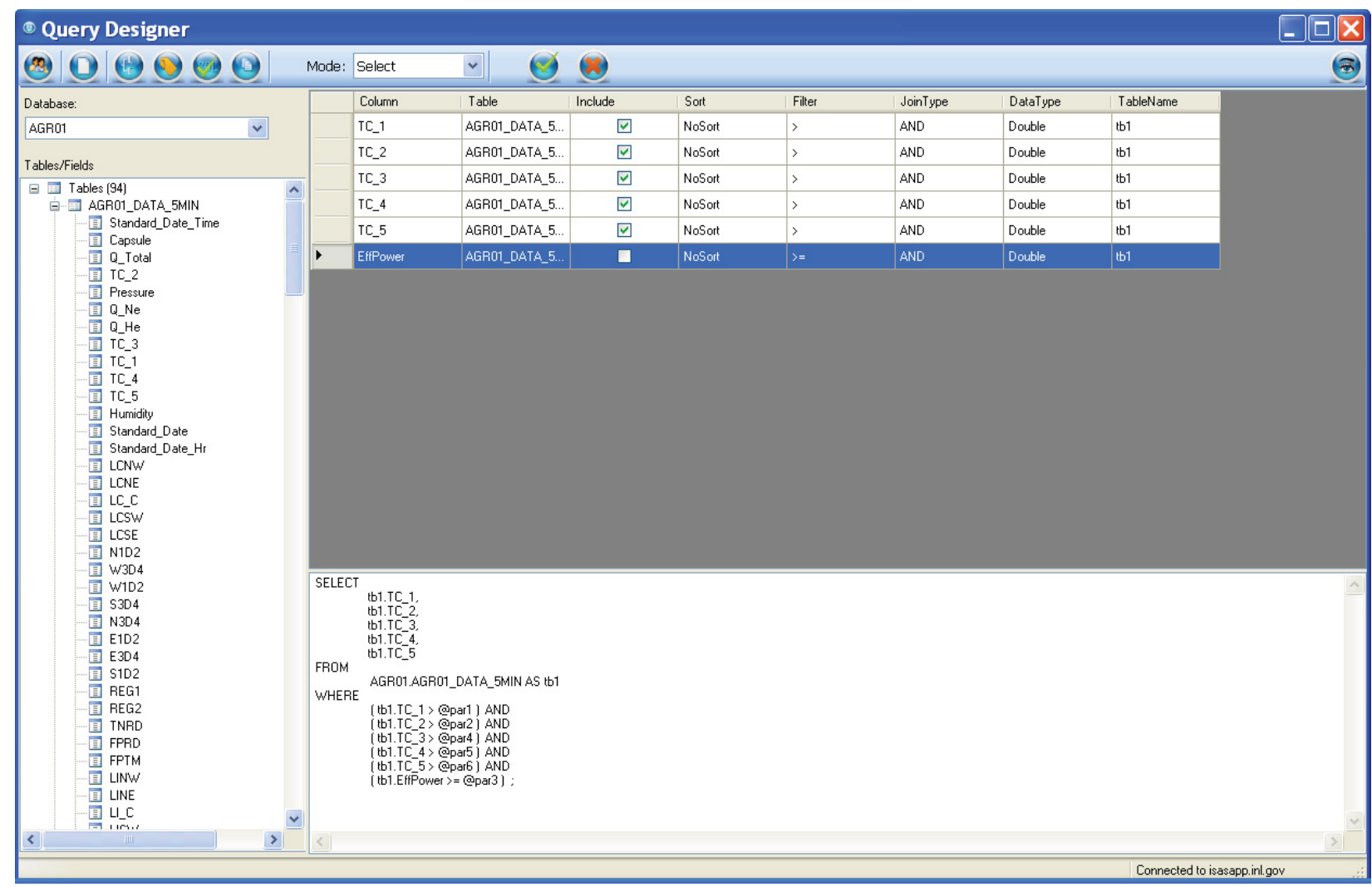

Figure 27. ModelCenter/SAS DataPipe plug-in for developing SQL queries connecting model inputs and outputs directly to the NDMAS repository.

Using ModelCenter will give NDMAS a method for collecting the analysis results from Methods model runs. This approach provides a direct method to maintain data provenance for Methods computational results. The project will be able to capture these model outputs in a controlled environment along with metadata to indicate the running environment for the models. 


\section{SUMMARY}

The overall goal of the NGNP NDMAS is to maintain data provenance for all NGNP data. This report describes management of the VHTR Methods data. The Lower Plenum Separate Effects study and a thermocouple prediction analysis is presented demonstrating basic capabilities available to manage Methods data streams.

NDMAS has multiple ways to access stored data. The main NDMAS Web portal environment allows users to access data, view the results of qualification tests, and view graphs and charts of various attributes of the data. Additional approaches are included for the management of the data output from VHTR simulation models and data generated from experiments designed to verify and validate the simulation codes. These simulation models represent the outcome of mathematical representation of VHTR components and systems. The Methods data management approaches described herein will handle data that arise from experiment, simulation, and external sources for the main purpose of facilitating parameter estimation and model V\&V.

The Lower Plenum Standard Problem data is available within NDMAS. The NDMAS Web portal currently offers access to the Lower Plenum data as a drillable data grid, associated graphs, and vector flow graphs. NDMAS also allows users to customize the presentation of the data by applying filters, conditional highlighting, and calculations of new quantities.

NDMAS captures data generated by NGNP models that perform simulations of reactor components and systems. Data derived from models complicate data provenance maintenance, since provenance is degraded when data is downloaded from a controlled database to personal computers (Excel, text, binary, etc.), fed as input into models, generated as results (Excel, text, binary, etc.), and then uploaded back into a database repository. Provenance in such an environment, if done at all, requires time-consuming hand entry methods. NDMAS has developed tools for maintaining provenance automatically during model execution, or data can be manually uploaded to NDMAS. 


\section{REFERENCES}

Buenman et.al., 2001, "Why and Where: A Characterization of Data Provenance. Lecture Notes in Computer Science," Volume 1973, International Conference on Database Theory (ICDT 2001), pages pp. 316-330.

Krusch, Susan E., 2009, PLN-2247, "General Software Management Plan for the VHTR TDO"

Krusch, Susan E., 2010, "VHTR TDO SMP Record Form," FRM-959-A, Rev 1, QL-2 Cust Dev, Used to Implement PLN-2247 for the NGNP Data Management and Analysis System (NDMAS), Version 2"

Schultz, R. R., 2005, Very High Temperature Reactor Design Methods Development and Validation Research \& Development Program Plan. INEEL/EXT-04-02293, Idaho National Laboratory, Idaho Falls, ID, 156 p. 
Appendix A

METHODS DATA QUALIFICATION GOALS 


\section{Appendix A}

\section{A-1. METHODS DATA QUALIFICATION GOALS}

The goals listed below were initially developed to stimulate thinking about Methods architecture.

These goals will be reviewed and updated, eventually becoming the basis of requirements for the Methods architecture and its integration into the Next Generation Nuclear Plant (NGNP) Data Management and Analysis System (NDMAS) Version 2.0. The main requirements are found in Schultz (2008).

1. Manage, to NQA-1 specifications (ASME 2000), the data output from very high temperature reactor (VHTR) simulation models and data generated from experiments designed to verify and validate the simulation codes.

2. Preserve the autonomy of the computational scientist, analyst, and experimentalist to use methods of their choosing for analysis of Methods Data:

a. Autonomy means the availability of tools and environments of their choosing, subject to their resource availability.

b. Limits on autonomy arise as needed for integration with other's work or when use by others is expected.

3. Provide a conduit to move data from personal workspaces to the NDMAS vault or other storage location.

a. NDMAS vault will provide an interface for this purpose. The interface provides input of metadata about the incoming data, whether experimental or simulated.

4. Provide a conduit to query and download experimental and simulated data, including the following approaches:
a. SAS portal
b. SAS Office plug-ins
c. SAS JMP
d. ModelCenter. ${ }^{\mathrm{a}}$

5. Provide a statistical analysis environment. Likely uses include:

a. Model parameter estimation

b. Comparison of model output to experimental data

c. Analysis of model uncertainty

d. Development of lower dimension statistical models based on data derived from higher dimension simulation experiments.

a. The software described here largely exists under the trade name ModelCenter ${ }^{\circledR}$, an established product of Phoenix Integration. ModelCenter has been used for engineering design work for over 15 years. One of its traditional uses is the conceptual design of aircraft and spacecraft, although it is important to note that it is completely neutral toward the type of application being integrated. To ModelCenter, an airplane or a watershed conservation strategy is the same, i.e., just a set of inputs, outputs, and a method to link and run the underlying codes. 
6. Store the results of model validation tests, including all pertinent information that would allow an independent third party to rerun the validation tests.

7. Provide a model coupling environment for construction of larger VHTR system models based on 'wrapping' existing and new codes that:

a. Does not require direct modification of codes

b. Provides for execution of these coupled models

c. Allows capture of the resulting output data

d. Provides for post simulation analysis

e. Allows display and access through a Web portal

f. Allows saving of coupled models as system models

g. Allows execution of system and wrapped models from a Web portal

h. Provides access to grid computing environment and models configured to available grids

\section{A-2. ARCHITECTURE}

Figure A-1 depicts the overall Methods data architecture that satisfies the goals listed in Section A-1. The architecture supports three main user groups; computational scientists (CSs), experimentalist/statistical analysts, and VHTR customers. 


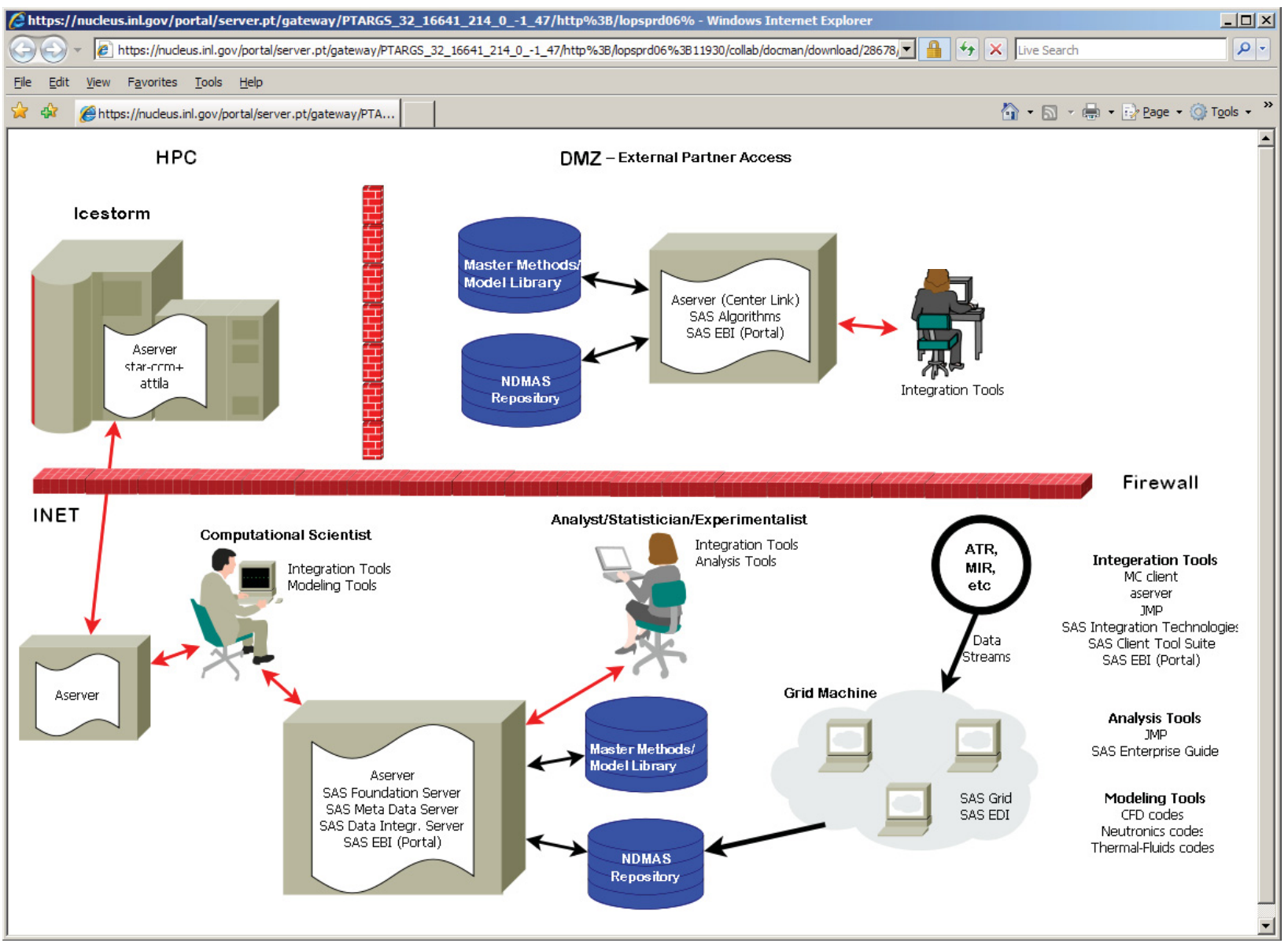

Figure A-1. Methods data architecture.

The architecture is divided into three main areas separated by Idaho National Laboratory (INL) firewalls. The INL intranet appears in the lower portion of Figure A-1. The term INET refers to the internal INL network. The INET is the main work network for VHTR Methods CSs, experimentalists, and statisticians. The high performance computing (HPC) enclave shown in upper left of Figure A-1 represents INL supercomputer capabilities. The INL Demilitarized Zone (DMZ) in the upper right of Figure A-1 is a computing area available through the internet from outside of INL. Overall, models and data transformed within the INL INET will be moved to the DMZ based on a review and approval process. This provides data access, report delivery, and selected model execution control to VHTR customers.

\section{A-2.1 INET Environment}

The INET contains most of the workstations used by the scientists to perform modeling and data analysis. The modeling and integration tools used by the scientists are a combination of desktop-based tools, databases and server-oriented tools and databases. This Methods architecture will provide linkage and sharing among desktop applications and servers. A specialized server will be used to communicate with the Icestorm supercomputer located in HPC enclave.

Data analysts and CSs can use their normal desktop setup to conduct work in their preferred configuration. If they need data or models from colleagues, Methods architecture has the ability to share 
data and models. Data can be shared using the NDMAS environment with access to VHTR Methods data found at http://isasapp.inl.gov/SASPortal. ${ }^{\mathrm{b}}$

In addition to VHTR data access, Methods architecture also has the capability to share individual models, explicitly couple shared models into larger scope system models, and deliver individual models and system models to the Web (both internally and externally on the DMZ). Models can be shared with colleagues by adding them to a model library in the 'Aserver' blocks, as shown in Figure A-1. Aserver is a Web server designed specially to provide remote access to models that have been "wrapped"c using tools provided by the Aserver software. Aserver can be installed on local desktop machines or on a remote server (see Model Repository in Figure A-1). The Aserver is configured to read data from the NDMAS data repository. Wrapped models directly access Methods experimental data. The Aserver also provides grid computing, since wrapped models executed on an individual's desktop use the computational resource of that desktop. This approach more closely controls the computational environment of particular models. A CS, who is a specialist in one particular field, can configure his/her desktop to run the model, then wrap the model for others to use, only exposing the inputs and outputs he/she feels are appropriate for certain user groups.

Models that run in the HPC enclave are configured to operate across the INET-HPC firewall. This serves two important purposes:

- The server manages the jobs that are submitted to Icestorm from the modeling and integration tools

- The server provides a single access point to Icestorm, thus minimizing the number of firewall rules necessary to accommodate the large number of scientists and their workstations.

This specialized server also provides a set of unique, world-class tools for use by the experimentalists and analysts. These tools provide such capabilities as statistical analysis, numerical analysis, what-if analysis, data cube creation and manipulation, data integration, data visualization, dashboards, etc.

Overall, the NDMAS and Methods Model Repository provides a centralized location for version control and configuration management of source code, test data, analysis results, and simulation results. The source code includes models, wrappers, scripts, etc. The repository will be integrated to the greatest extent possible with the tools used by the scientists, experimentalists, and analysts.

Once models have been tested and verified, they can be submitted to the Model Library. The library organizes the models, captures metadata that describe the nature and capabilities of the models, and stores the wrappers and other modules necessary for integration. The integration tools used by scientists can then be connected to this library, giving the scientists a broad selection of models to choose from as they integrate and then execute multiple models of various forms for various purposes.

The analysis tools can be computationally demanding, especially when applied to the large data sets that are anticipated to be available to the experimentalists and analysts. It is therefore necessary to provide specialized computing capabilities so that these tools can perform their computations in a reasonable amount of time. To meet this need, a Grid ${ }^{\mathrm{d}}$ computing capability will be available to the end users of the analysis tools. The grid is managed by SAS software, which distributes the computations over a set of compute nodes. The analysis tools can then communicate directly with the grid software, thus hiding the complexity of the grid from end users.

Data streams are produced by experiments and simulations and stored on the grid storage array. Since those data streams are anticipated to be relatively large, they will be stored using a specialized file system

b, Send an email to Dave McGrath (david.mcgrath@inl.gov) or Leng Vang (leng.vang@inl.gov) for access instructions.

c. "Wrapping" is a term for an interface that surrounds an executable computer program (i.e., a VHTR simulation model) and allows inputs and outputs to be exchanged between and external system model and the wrapped code.

d. In the context of this architecture, Grid refers to the SAS Grid Computing architecture. 
that sits on top of the native file systems and provides robust, high-speed storage and retrieval for these large data sets.

\section{A-2.2 INL HPC Environment}

The INL HPC environment (see Figure A-1) consists of several supercomputers, Icestorm being the most powerful. Icestorm is used in this architectural discussion to represent any or all of the computational capability that exists within the INL's HPC environment. Within the context of this architecture, Icestorm is used to execute models and simulations for the scientists.

Icestorm is located in a security enclave, known as the "HPC enclave," that separates it from other computational resources at the INL. The separation is represented by the red brick "firewalls" in Figure A-1. Users are able to access Icestorm by being located within the HPC enclave (directly or via a virtual private network), or by accessing it through a firewall opening created for that purpose. Firewall openings are created by means of a firewall rule that allows such access. However, the firewall rules used to allow access through firewalls are typically based on the "one to one" security model. This means that each rule that is applied to the firewall must precisely identify the two specific machines (one on each side of the firewall) that need to communicate with each other. The firewall rules also describe the nature of the communication that needs to occur. Accordingly, the number of such firewall rules must be kept to a minimum in order to keep the rule set manageable and to minimize the number of openings in the firewall itself.

\section{A-2.3 INL DMZ Environment}

The DMZ at INL is a public-facing network, and is separated from the other networks and enclaves by a firewall. Because of the high degree of security risks present in a public-facing network, computing resources in the DMZ are prevented from directly communicating with resources in other INL enclaves. However, a significant portion of the objectives of this initiative for end users requires that a subset of the overall capabilities be available to VHTR customers that are located outside of the INL firewall. Therefore, this subset of capabilities has been identified and will be made available in the DMZ. The servers that provide these capabilities will essentially be isolated from the other servers described above. The capabilities will focus on the model integration and business intelligence described in the applicable use case scenarios in Section A-1.1.4 below.

\section{A-2.4 Current NDMAS Version 1.1. Repository}

The current NDMAS Version 1.1 architecture shown in Figure A-2 depicts data moved from source files into a secure data vault and archived as discussed in (Section 6). Data can then be extracted from the vault and analyzed (staged in Figure A-2) or delivered to customers and colleagues (delivery in

Figure A-2). 


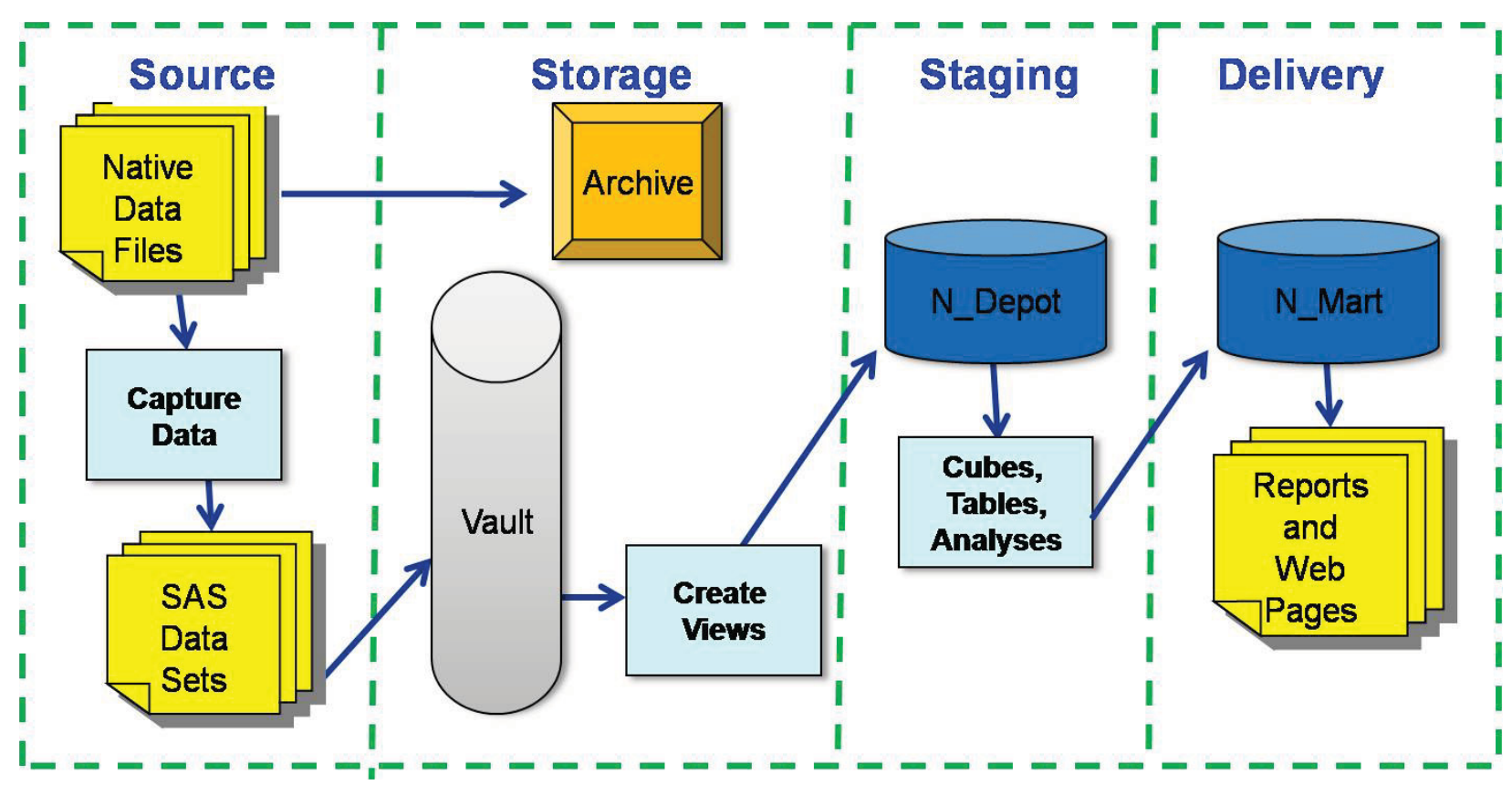

Figure A-2. NDMAS Version 1.1 data storage architecture.

\section{A-3. MODELCENTER OVERVIEW}

\section{A-3.1 National Aeronautics and Space Administration}

Phoenix Integration has been providing software technologies and solutions to National Aeronautics and Space Administration (NASA) for over 10 years. Six NASA centers actively use Phoenix Integration products (Langley, Glenn, Kennedy, Marshall, JPL, and Johnson). Phoenix Integration's CenterLink (distributed computing) and Data Visualization (decision support) products both derive from successful NASA Phase II SBIR research projects. Phoenix Integration is currently working on a NASA Langley sponsored Phase II SBIR project (NNL08AA09C) to extend existing ModelCenter capabilities for MultiDisciplinary Analysis and Optimization (MDAO). Phoenix Integration also recently completed a NASA (JPL) sponsored Phase II SBIR project (NNC06CA29C) to develop a human-in-the-loop environment for conceptual and preliminary engineering design. The resulting decision support environment helps designers generate engineering data, compare design alternatives, understand key trends, and communicate results to colleagues and management. It is the basis, in fact, of the decision support tools proposed here. Indeed, NASA sponsored research is central to ModelCenter's widespread applicability, far outside the aerospace domain.

\section{A-3.2 Lockheed Martin and Boeing}

Lockheed Martin and Boeing represent two of Phoenix Integration's largest customers. Lockheed Martin engineers use ModelCenter across a wide range of different projects, such as DARPA's Quiet Supersonic Platform, to rapidly create custom design processes linking dozens of specialized engineering applications running from the company's global enterprise. At Boeing, Phoenix Integration software was chosen to form the foundation for the Boeing Integrated Vehicle Design System, a framework enabling model and data integration across three different facilities across the country. In addition, Phoenix Integration maintains a product development relationship with Boeing for exclusive commercial distribution of Design Explorer, a critical part of the Phoenix optimization platform. Design Explorer has been used on numerous projects, including the power supply for the International Space Station. 
Although the examples cited above are aerospace related, Phoenix Integration software is completely generic in terms of which application it is used for. The company has a wide variety of customers (e.g., General Motors, Caterpillar, and Dresser-Rand) and has produced case studies to demonstrate applicability in many areas. One such study was the design and economics of a delivery system for plant air to an industrial facility. All components of the system were integrated including a turbo-compressor, pressure vessel, heat exchangers, and a wind turbine to supply the needed power. Many of the components in this model were themselves ModelCenter models, demonstrating the feasibility of building larger system models from subsystem components. One such subsystem was a wind farm model where the National Renewable Energy Laboratory (NREL) supplied spreadsheets were integrated to size the wind turbines. This study is particularly relevant to the situation here, since many disparate models were integrated, the focus was economic performance of a proposed investment, and a large system was studied. The final result was a Pareto curve of possibilities where a customer could choose the best performing system at various price points.

Despite its well-proven capability, ModelCenter lacks the ability to link directly to the NDMAS repository with resorting to script level programming. 


\section{A-4. SCRIPT CODE TO ACCESS NDMAS REPOSITORY}

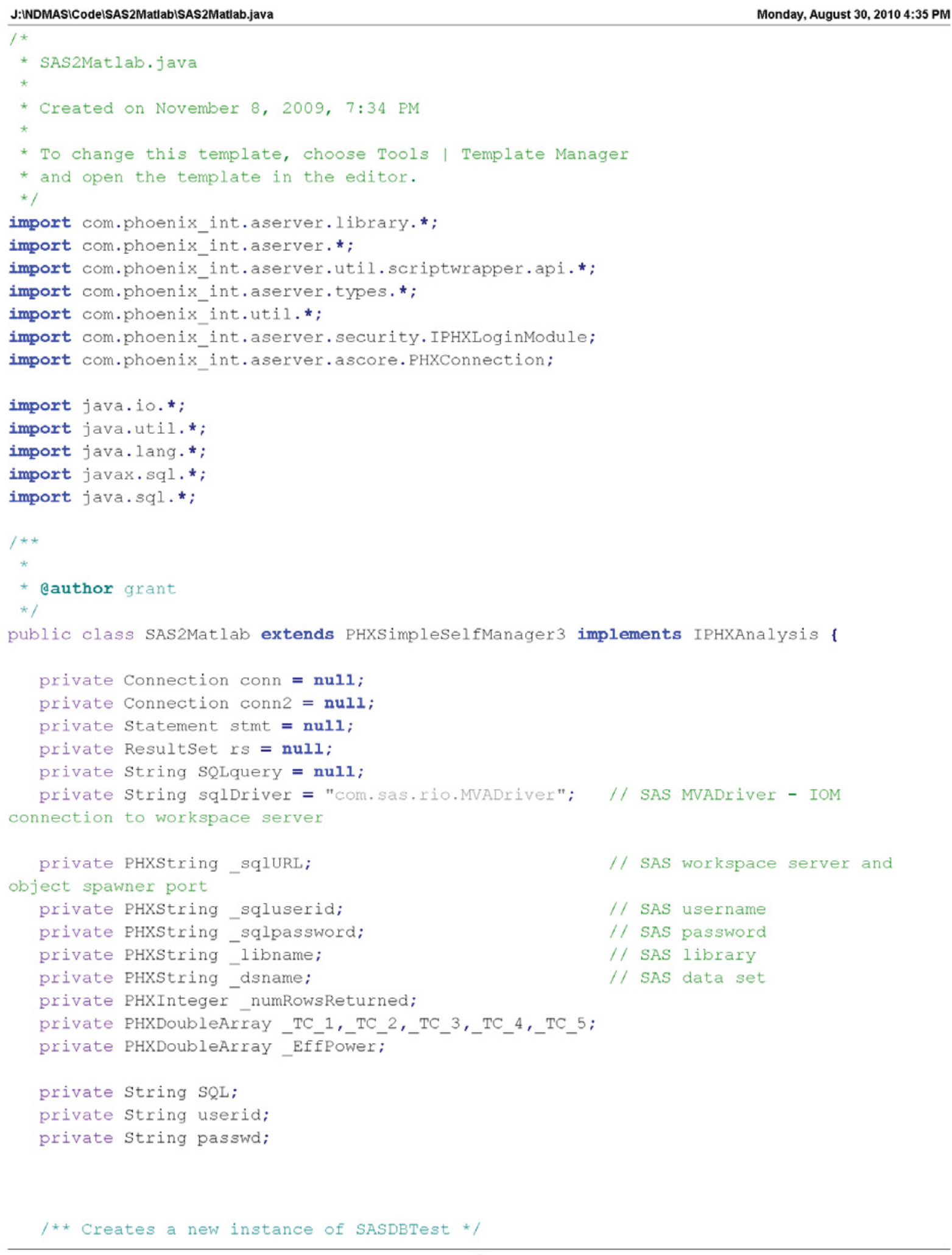


public SAS2Matlab() \{

try

1

sqlURL $=$ new PHXString () ;

// Port 8561 is Meta Data Server;

// Port 8591 is Work Space Server

//_sqlURL.setValue( "jdbc:sasiom://sasngnp.inl.gov:8561" );

_sqlURL.setvalue( "jdbc:sasiom://isasapp.inl.gov:8591") ;

addVariable("sqluRL", true, _sqluRL);

libname $=$ new PHXString ();

_ibname.setValue ( "AGR01" );

addVariable( "libName", true, _libname);

dsname $=$ new PHXString ();

_dsname.setvalue ( "AGR01 DATA 5MIN");

addVariable( "datasetName", true,_dsname);

// Allocate arrays

int size $=143770$;

_TC_1 = new PHXDoubleArray ();

TC 2 = new PHXDoubleArray ();

TC 3 = new PHXDoubleArray ();

TC 4 = new PHXDoubleArray ();

-TC_5 = new PHXDoubleArray ();

_EffPower = new PHXDoubleArray ();

- numRowsReturned = new PHXInteger ();

numRowsReturned. setValue (-1):

addVariable ( "TC 1", false, TC 1);

addVariable ( "TC 2", false, TC_2);

addVariable ( "TC 3", false, _TC_3);

addVariable( "TC_4", false, _TC_4);

addVariable( "TC_5", false, _TC_5);

addVariable( "EffPower", false, EffPower);

addVariable( "numRows", false, numRowsReturned);

\}

//catch (java.sql.SQLException e) (

// e.printstackTrace() ;

//) catch (ClassNotFoundException e) I

// e.printstackTrace() ;

// ]

catch (Exception e)

\{

System.out.println (SQL);

e.printstackTrace ();

\} 


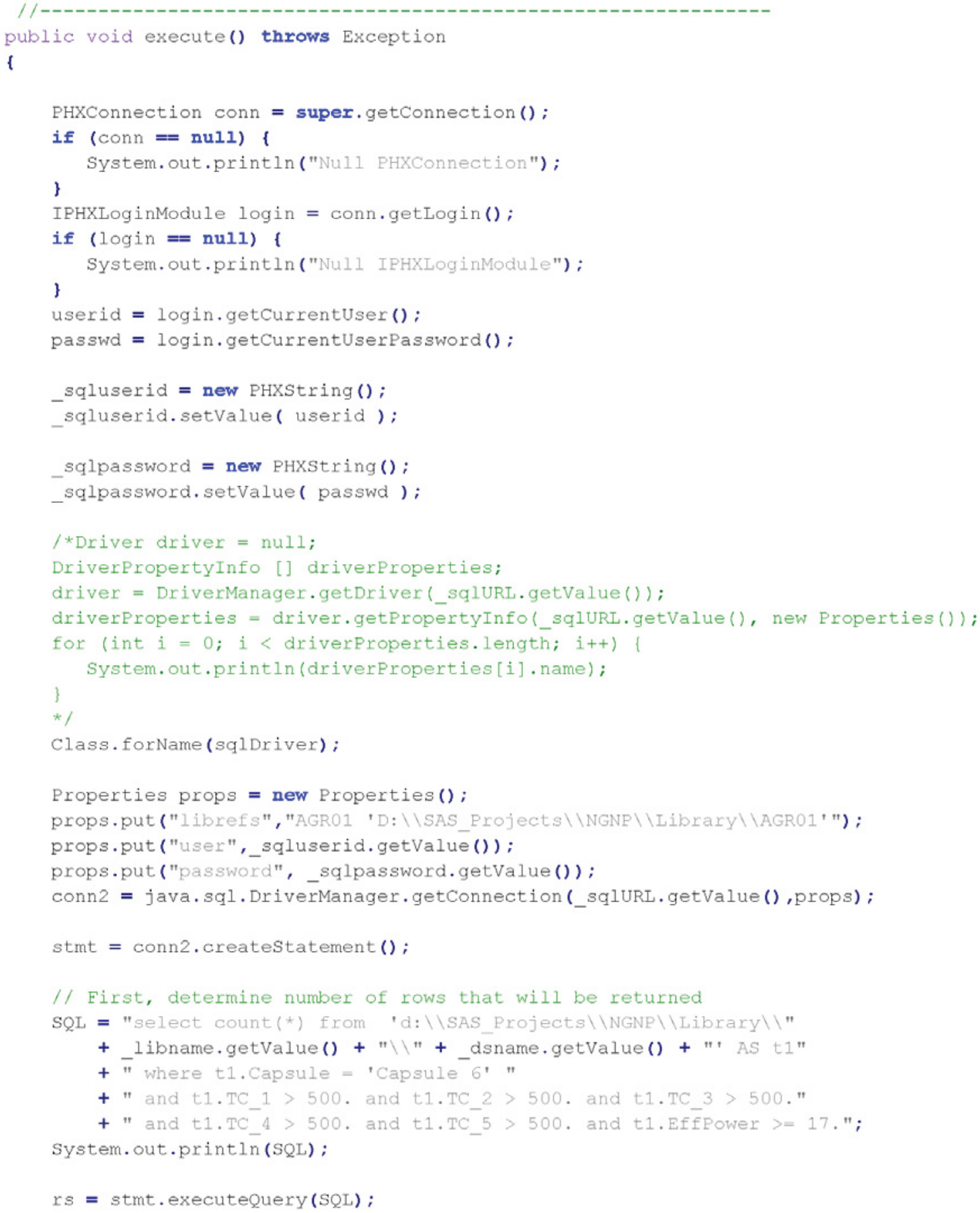




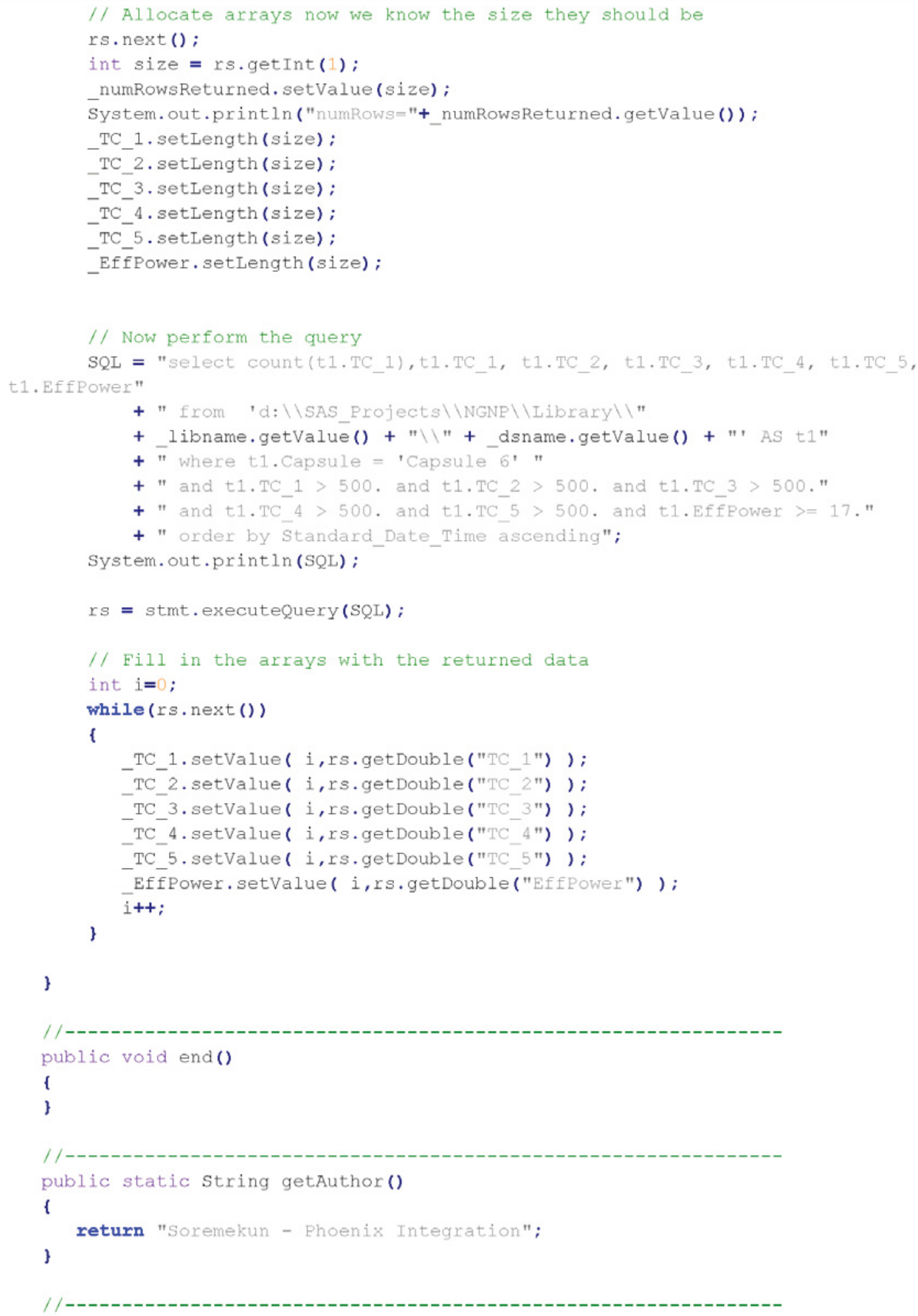


public static string geticonfile()

return $" n$;

\}

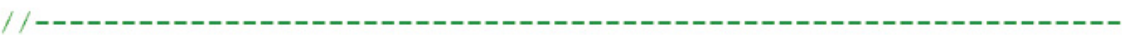

public static string getversion() \{

return "0.1, 11/2009";

\}

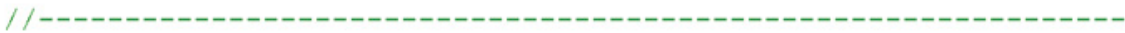

public static string getDescription()

\{

return "Wrapper for comminucating with a SAS Web Service / table

(http://mcgrdo9.inl.gov/ECos/PriceCarbonEu.cfc?wsdl) that returns the price of carbon"; \}

\} 


\section{A-5. REFERENCES}

ASME, 2000, “Quality Assurance Requirements for Nuclear Facility Applications," Nuclear Quality Assurance (NQA-1), ASME International, New York, NY.

Schultz, R. R., 2008, Next Generation Nuclear Plan Methods Research and Development Technical Program Plan, PLN-2498, Idaho National Laboratory, Idaho Falls, ID, 203 p. 